\title{
Water wave scattering by impermeable and perforated plates
}

\author{
Liang, Hui; Zheng, Siming; Shao, Yanlin; Hian Chua, Kie ; Sang Choo, Yoo ; Greaves, Deborah
}

Published in:

Physics of Fluids

Link to article, DOI:

$10.1063 / 5.0051355$

Publication date:

2021

Document Version

Peer reviewed version

Link back to DTU Orbit

Citation (APA):

Liang, H., Zheng, S., Shao, Y., Hian Chua, K., Sang Choo, Y., \& Greaves, D. (2021). Water wave scattering by impermeable and perforated plates. Physics of Fluids, 33(7), [077111]. https://doi.org/10.1063/5.0051355

\section{General rights}

Copyright and moral rights for the publications made accessible in the public portal are retained by the authors and/or other copyright owners and it is a condition of accessing publications that users recognise and abide by the legal requirements associated with these rights.

- Users may download and print one copy of any publication from the public portal for the purpose of private study or research.

- You may not further distribute the material or use it for any profit-making activity or commercial gain

- You may freely distribute the URL identifying the publication in the public portal

If you believe that this document breaches copyright please contact us providing details, and we will remove access to the work immediately and investigate your claim 


\title{
Water wave scattering by impermeable and perforated plates
}

\author{
Hui Liang (梁辉), ${ }^{1,}$ a) Siming Zheng (郑思明), ${ }^{2,3, b)}$ Yanlin Shao (邵炎林) ${ }^{4},{ }^{4}$ Kie Hian Chua (蔡棋轩), ${ }^{1}$ Yoo \\ Sang Choo (曹耀生), ${ }^{1}$ and Deborah Greaves ${ }^{3}$ \\ 1) Technology Centre for Offshore and Marine, Singapore (TCOMS), 118411, \\ Singapore \\ ${ }^{2)}$ State Key Laboratory of Hydroscience and Engineering, Tsinghua University, Beijing, 100084, \\ China \\ 3) School of Engineering, Computing and Mathematics, University of Plymouth, Drake Circus, Plymouth PL4 8AA, \\ $U K$ \\ ${ }^{4)}$ Department of Mechanical Engineering, Technical University of Denmark, 2800 Lyngby, \\ Denmark \\ ${ }^{5)}$ College of Shipbuilding Engineering, Harbin Engineering University, Harbin, 150001, \\ China
}

(Dated: 3 July 2021)

\begin{abstract}
In the field of offshore renewable energy, impermeable plates are used as underwater lenses to amplify the wave amplitude, and perforated plates can harness wave energy as a power take-off device. Within the framework of the linear potential-flow theory, the water wave scattering by impermeable and perforated horizontal plates is investigated in the present study, and both circular and elliptical plates are considered. The hypersingular integral equation is constructed to model the interaction between water waves and plates of small thickness. For wave scattering by impermeable plates with the focus on wave amplification, wave interference effects due to multiple plates can be utilized to achieve large wave amplification. For perforated plates used for harnessing wave energy, deploying an array of elliptical plates is promising if the deployment line coincides with the major axis and the incident wave propagates along the minor axis. The study gives an insight into harnessing energy from water waves by horizontal plates.
\end{abstract}

Keywords: Hypersingular integral equation; Perforated plate; Wave amplification; Wave energy

\section{INTRODUCTION}

Impermeable and perforated plates are omnipresent in a wide range of engineering applications. In marine engineering, thin plates are attached to Spar platforms to provide extra added mass and damping so that violent wave-frequency heave resonance is avoided (Molin, 2011). Fixed and floating breakwaters are usually equipped with perforated plates to attenuate waves in coastal engineering (Huang, Li, and Liu, 2011; Behera and Sahoo, 2015; Selvan et al., 2021). In offshore renewable energy, piezoelectric and perforated disks (Renzi, 2016; Meylan, Bennetts, and Peter, 2017; Zheng et al., 2020b,a) as well as flap-type wave energy converters (WECs) (Renzi and Dias, 2012; Sarkar, Renzi, and Dias, 2014) have huge potential for wave-power extraction. Moreover, impermeable plate is usually used as a 'wave lens' to focus waves so that wave energy is amplified (Stamnes et al., 1983; Newman, 2015), and perforated plate can be adopted to simulate the performance of plate-shaped WECs, provided that a special power take-off (PTO) system is designed (Meylan, Bennetts, and Peter, 2017; Zheng et al., $2020 \mathrm{a}, \mathrm{b})$. In addition to the artificial structures as mentioned above, there are also some plate-shaped structures in the nature, e.g., sea ice and stingrays, the hydrodynamics of which is of significant interest for engineering

\footnotetext{
a)Electronic mail: liang_hui@tcoms.sg

b) Electronic mail: siming.zheng@plymouth.ac.uk
}

and biology (e.g., see Meylan et al., 2015; Li, Wu, and Ji, 2018; Li, Wu, and Shi, 2018; Bottom II et al., 2016).

In the context of wave-structure interactions, the boundary integral method is widely applied, and great success has been witnessed in fields of marine engineering, coastal engineering, and offshore renewable energy, etc. However, the direct application of a boundary integral equation to deal with plates of vanishing thickness is problematic, and the coefficient matrix may even be illconditioned. The reason is that two opposite panels on the side surfaces of a shell structure yield almost the same influence coefficients resulting in parallel vectors. As a consequence, the coefficient matrix will be ill-conditioned with a large condition number, leading to loss of accuracy of the numerical solutions. As a compromise, one may also model the thin plate as finite thickness to avoid parallel vectors, which however requires distribution of singularities over all surfaces of the plate, thus unnecessarily increasing the number of unknowns and computational costs.

To resolve this issue, an alternative is the application of the multi-domain boundary element method (Mackay, Liang, and Johanning, 2021), but this method is limited to the scenario that the perforated surface together with a free surface enclose a bounded fluid region. Therefore, a general treatment is to model the flow induced by a structure of small thickness with a dipole distribution and consider the velocity potential jump across the structure (Parsons and Martin, 1992; Martin and Farina, 1997). The modeling of a dipole distribution 
gives rise to a hypersingular integral equation, which requires careful treatment in the numerical implementation (Hariri Nokob and Yeung, 2015).

The hypersingular integral equation originally developed in solid mechanics was brought to solve the wavestructure interaction problems by Parsons and Martin (1992) and Martin and Farina (1997). In the 21st century, the hypersingular integral equation has been widely applied in applications, such as: floating harbors, and offshore renewable energy (Renzi and Dias, 2012, Hariri Nokob and Yeung, 2015, Porter, 2015). It has been demonstrated that the hypersingular integral equation can deal with thin shell structures very well. However, the existing work on hypersingular integral equations (Renzi and Dias, 2012, Hariri Nokob and Yeung, 2015, Porter, 2015) is tailored to specific problems. The objective of the present study is to develop a general method applicable to structures of arbitrary geometry.

In the present study, the linear interaction between water waves and impermeable as well as perforated plates is investigated by a general hypersingular integral equation. Despite diverse usefulness of impermeable and perforated plates, we focus on the wave amplification by impermeable plates and wave energy absorption by perforated plates. While the single circular plate has been are extensively studied in the literature (Martin and Farina, 1997; Porter, 2015), multiple circular plates are considered in this paper. Unlike the work in Zheng et al. (2020a,b), the present approach is more general and capable of analyzing multiple plates with staggered arrangement with horizontal overlapping. In addition to circular plates, the hydrodynamic performance of elliptical plates, which has been rarely studied in the literature, is also investigated. The layout of the paper is as follows.

In Sect. II, the basic equations, including: the hypersingular integral equation and boundary conditions, are formulated. A method to deal with interactions between water waves and submerged impermeable/perforated plates is developed. The verification of the developed numerical method is conducted in Sect. III via comparing the numerical solutions with the existing benchmark results documented in the literature. In Sect. IV, wave scattering by impermeable plates is studied with the focus on the wave amplification. Large wave amplification can be achieved by deploying an array of impermeable plates making use of wave interference effects. In Sect. V, wave scattering by perforated plates is studied, and the focus is placed on wave energy absorption. Recommendations for efficiently harnessing wave energy are given. Lastly, concluding remarks and future perspectives are presented in Sect. VI.

\section{BASIC EQUATIONS}

Basic equations and boundary conditions within the context of linear potential-flow theory are now formulated. Despite limitations of small wave steepness, the applicability of the linear potential-flow theory has been well justified in engineering practice (see Mei, Stiassnie, and Yue, 2017, Chapt. 8).

A three-dimensional Cartesian system of coordinates $O x y z$ is defined with the $O x y$ plane coinciding with the undisturbed free surface and $\mathrm{Oz}$ axis pointing positively upward. In the study of wave-structure interactions in which the inertial effect is predominant (Faltinsen, 1993; Molin, 2002), it is usually assumed that the fluid is inviscid and incompressible, and the flow is irrotational so that a velocity potential $\Phi$, satisfying $\nabla^{2} \Phi=0$ in a fluid domain of infinite lateral extent and water depth $d$, exists. Under an assumption of small wave steepness, the problem can be linearized, and the validity of the linear model to deal with wave scattering by horizontal plates has been demonstrated in Zheng et al. (2020b).

In a steady state, the velocity potential is written as $\Phi(x, y, z, t)=\operatorname{Re}\left[\phi(x, y, z) \mathrm{e}^{-\mathrm{i} \omega t}\right]$, where $t$ denotes time and $\omega$ means the angular frequency of oscillation. For the wave scattering problem, the total potential in the flow field, diffraction potential $\phi_{\mathrm{D}}$, can be decomposed into an incident wave potential $\phi_{\mathrm{I}}$ and a scattering potential $\phi_{\mathrm{S}}$, i.e.: $\phi_{\mathrm{D}}=\phi_{\mathrm{I}}+\phi_{\mathrm{S}}$. The boundary-value problem for the scattering potential $\phi_{\mathrm{S}}$ is written as

$$
\begin{array}{lr}
\nabla^{2} \phi_{\mathrm{S}}=0 & \text { in the fluid domain, } \\
-\omega^{2} \phi_{\mathrm{S}}+g \frac{\partial \phi_{\mathrm{S}}}{\partial z}=0 & \text { on } z=0, \\
\frac{\partial \phi_{\mathrm{S}}}{\partial z}=0 & (1 \mathrm{~b}) \\
& \text { on } z=-d,
\end{array}
$$

$$
\begin{aligned}
& \lim _{R \rightarrow \infty} \sqrt{R}\left(\frac{\partial \phi_{\mathrm{S}}}{\partial R}-\mathrm{i} k_{0} \phi_{\mathrm{S}}\right)=0 \\
& \frac{\partial \phi_{\mathrm{S}}}{\partial n}=-\frac{\partial \phi_{\mathrm{I}}}{\partial n}-\mathrm{i} \sigma\left(\phi^{+}-\phi^{-}\right)
\end{aligned}
$$

in the far field,

on $S$.

Equations (1a) - (1e) correspond to the Laplace equation, linearized free-surface boundary condition, bottom condition, far-field radiation condition, and body boundary condition, respectively. $g$ denotes the gravitational acceleration, $n$ the vector normal to the structure, $S$ the plate surface, $\phi^{+}$and $\phi^{-}$the velocity potential on the upper and lower sides of the plates, and $\phi_{\mathrm{I}}$ the velocity potential of incident waves expressed as (Newman, 1977):

$$
\phi_{\mathrm{I}}=-\mathrm{i} \frac{a g}{\omega} \frac{\cosh k_{0}(z+d)}{\cosh k_{0} d} \mathrm{e}^{\mathrm{i} k_{0}(x \cos \beta+y \sin \beta)},
$$

where $k_{0}$ denotes the wavenumber in finite water depth satisfying $\omega^{2}=g k_{0} \tanh k_{0} d, a$ is the wave amplitude, and $\beta$ means the wave heading. In the body boundary condition (1e), $\sigma$ represents a porosity parameter, which may be expressed as (Chwang, 1983; Chwang and Chan, 
1998):

$$
\sigma=\frac{b k_{0}}{2 \pi},
$$

where $b$ is a nondimensional parameter associated with the solidity ratio. Here, both impermeable and perforated plates are allowed for. When the plate surface is impermeable, $\sigma$ is null.

To solve the Laplace equation, a boundary integral equation is adopted. According to Parsons and Martin (1992) and Martin and Farina (1997), the flow induced by a plate of small thickness can be represented by a dipole distribution

$$
-4 \pi \phi_{\mathrm{S}}(\boldsymbol{x})=\int_{S} \psi(\boldsymbol{\xi}) \frac{\partial G(\boldsymbol{x}, \boldsymbol{\xi})}{\partial n_{\boldsymbol{\xi}}} \mathrm{d} S,
$$

where $\boldsymbol{x} \equiv(x, y, z)$ and $\boldsymbol{\xi} \equiv\left(x_{0}, y_{0}, z_{0}\right)$ denote the flowfield point and singularity point, respectively, and $\psi=$ $\phi^{+}-\phi^{-}$stands for the velocity potential jump across the plate surface. The free-surface Green function $G$, satisfying all conditions in (1) except the body boundary condition (1e), is expressed as (Wehausen and Laitone, 1960)

$$
G=-\frac{1}{r}-\frac{1}{r^{\prime}}+G^{F}
$$

where

$$
r=\sqrt{\mathcal{R}^{2}+\left(z-z_{0}\right)^{2}} \text { and } r^{\prime}=\sqrt{\mathcal{R}^{2}+\left(z+z_{0}+2 d\right)^{2}}
$$

with $\mathcal{R}=\sqrt{\left(x-x_{0}\right)^{2}+\left(y-y_{0}\right)^{2}}$, and the free-surface term $G^{F}$ in finite water depth is in the form of a single wavenumber integral

$G^{F}=-2 \int_{0}^{\infty} \frac{(k+K) \cosh k(z+d) \cosh k\left(z_{0}+d\right)}{(k \sinh k d-K \cosh k d) \mathrm{e}^{k d}} J_{0}(k \mathcal{R}) \mathrm{d} k$,

where $K=\omega^{2} / g$ is the wavenumber in deep water, and $J_{0}$ denotes the zeroth-order Bessel function of the first kind. The integral in Eq. (5c) is interpreted as bypssing the pole at $k=k_{0}$ from below so that the radiation condition is satisfied. The evaluation of the free-surface term $G^{F}$ in Eq. (5c) has been extensively studied in the literature (Newman, 1985, Chen, 1993, Liu et al., 2020, Mackay, 2019). In the present study, the free-surface term is evaluated using the algorithm described by Newman (1985).

To determine the velocity potential jump $\psi(\boldsymbol{\xi})$, we take the normal derivative of the integral equation (4) resulting in a hypersingular integral equation on the body surface:

$$
\iint_{S} \psi(\boldsymbol{\xi}) \frac{\partial^{2} G(\boldsymbol{x}, \boldsymbol{\xi})}{\partial n_{\boldsymbol{x}} \partial n_{\boldsymbol{\xi}}} \mathrm{d} S=4 \pi\left[\frac{\partial \phi_{\mathrm{I}}(\boldsymbol{x})}{\partial n}+\mathrm{i} \sigma \psi(\boldsymbol{x})\right],
$$

where the body boundary condition $(5 \mathrm{c})$ is recalled. Discretization of the plate surface yields a finite number quadrilateral panels. Over each panel, the velocity potential jump is assumed constant, and then the discretized hypersingular integral equation is written in the form of

$$
[\boldsymbol{A}]\{\boldsymbol{\psi}\}=\{\boldsymbol{b}\},
$$

in which the elements of the coefficient matrix $[\boldsymbol{A}]$ are expressed as

$$
A_{i, j}=\iint_{S_{j}} \frac{\partial^{2} G(\boldsymbol{x}, \boldsymbol{\xi})}{\partial n_{\boldsymbol{x}} \partial n_{\boldsymbol{\xi}}} \mathrm{d} S=A_{i, j}^{R}+A_{i, j}^{F} .
$$

Here $A_{i, j}^{R}$ and $A_{i, j}^{F}$ correspond to the Rankine and freesurface terms in the free-surface Green function, which are written as

$$
\begin{gathered}
A_{i, j}^{R}=\iint_{S_{j}} \frac{\partial^{2}}{\partial n_{\boldsymbol{x}} \partial n_{\boldsymbol{\xi}}}\left(-\frac{1}{r}-\frac{1}{r^{\prime}}\right) \mathrm{d} S, \\
A_{i, j}^{F}=\iint_{S_{j}} \frac{\partial^{2} G^{F}(\boldsymbol{x}, \boldsymbol{\xi})}{\partial n_{\boldsymbol{x}} \partial n_{\boldsymbol{\xi}}} \mathrm{d} S .
\end{gathered}
$$

The hypersingular feature stems from the calculation of the normal velocity induced by a dipole distribution as in Eq. (8a), which requires special attention in the numerical implementation. This difficulty has been recently circumvented in Liang, Shao, and Chen (2021). The uniform dipole distribution can be transformed to a vortex ring comprising edge segments of the panel, and the induced velocity components are then obtained by the Biot-Savart law. For the integration of second derivatives of the free-surface term $G^{F}$ over a panel, the numerical quadrature rule has been applied.

Given the velocity potential jump $\psi$ determined by the hypersingular integral equation (4), the wave force acting on structures can be obtained

$$
\boldsymbol{F}=-\mathrm{i} \omega \rho \iint_{S} \psi(\boldsymbol{x}) \boldsymbol{n} \mathrm{d} S
$$

where $\rho$ represents the water density. Based on the dynamic free-surface boundary condition, the scattered free-surface wave elevation is obtained:

$$
\mathcal{E}_{\mathrm{S}}=\frac{\mathrm{i} \omega}{g} \phi_{\mathrm{S}}(x, y, 0),
$$

and the free-surface elevation of diffracted waves $\mathcal{E}$ comprising of incident waves $\mathcal{E}_{\mathrm{I}}$ and scattered waves $\mathcal{E}_{\mathrm{S}}$ is then written as

$$
\mathcal{E}=\mathcal{E}_{\mathrm{I}}+\mathcal{E}_{\mathrm{S}} \text { with } \mathcal{E}_{\mathrm{I}}=a \mathrm{e}^{\mathrm{i} k_{0}(x \cos \beta+y \sin \beta)} .
$$

When the plate is perforated, it can be used as a PTO device to harness wave power. Following Zheng et al. $(2020 \mathrm{a}, \mathrm{b})$, the energy absorbed by a submerged perforated plate is expressed as:

$$
P_{\mathrm{diss}}=\frac{\rho \omega}{2} \sum_{n=1}^{N} \sigma_{n} \iint_{S_{n}}\|\psi\|^{2} \mathrm{~d} S
$$


where $S_{n}$ and $\sigma_{n}$ denote the area and the corresponding perforation parameter of panel $n$. Given the incident wave energy per unit width (Newman, 1977)

$$
P_{\text {in }}=\frac{\rho g a^{2}}{2} \frac{\omega}{2 k_{0}}\left(1+\frac{2 k_{0} d}{\sinh 2 k_{0} d}\right),
$$

the nondimensional absorbed wave energy is written as

$$
\chi_{\text {diss }}=k_{0} P_{\text {diss }} / P_{\text {in }} .
$$

\section{VERIFICATION OF THE NUMERICAL METHOD}

Verification of the developed numerical method is now considered via comparing with the existing benchmark results.

The convergence test is first conducted via calculating the wave exciting force by an impermeable circular plate of a radius $R$. The normalized submergence of the plate is $v / R=0.2$, and the water depth is $d / R=2.0$, where $v$ denotes the submergence depth of the plate. Three different mesh resolutions are considered, which correspond to 800, 3200, and 12800 quadrilateral panels, and are identified as 'Coarse', 'Medium', and 'Fine', respectively. Fig. 1 depicts the wave exciting forces on the impermeable circular plate as a function of normalized wavenumber $k_{0} R$, and comparison is made with the analytical solution determined by the method of eigenfunction expansion in Zheng et al. (2020a). The vertical wave exciting force is nondimensionalized with respect to $\pi \rho g a R^{2}$. It is observed that the results associated with 'Medium' and 'Fine' mesh resolutions are in good agreement, whereas the difference at the peak from those with 'Coarse mesh' is appreciable. Moreover, the results associated with 'Medium mesh' and 'Fine mesh' agree well with the analytical solution. It indicates that the 'Medium mesh' can yield converged and accurate results, and this mesh density will be used hereafter.

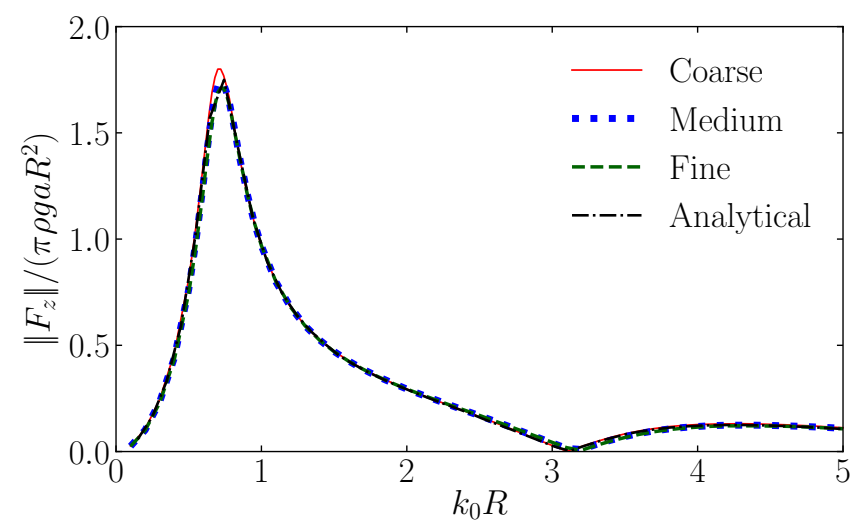

FIG. 1: Nondimensional wave exciting forces for three different mesh resolutions for $v / R=0.2$, and $d / R=2.0$.

To verify the method applied to perforated plates, the wave power absorption by a single perforated plate is con- sidered. Fig. 2 depicts the nondimensional wave energy defined in Eq. (14) absorbed by a single circular plate for different submergence depths, inducing: $v / R=0.2$, 0.5 , and 1.0 as shown in subplots (a), (b), and (c), respectively. The water depth is $d / R=2.0$. Generally, the smaller the immersion depth is, the higher wave power is harnessed. The reason is that the wave kinematics decay with the immersion depth. Comparison is made with the analytical solution by the method of eigenfunction expansion described in Zheng et al. (2020a), and the agreement is satisfactory. Therefore, the developed numerical method is verified.

\section{IMPERMEABLE PLATES}

Wave scattering by impermeable circular and elliptical plates is now considered. The elliptical plate is sketched in Fig. 3, the semi-major and semi-minor axes are denoted by $R_{1}$ and $R_{2}$, respectively. Here, we define the aspect ratio of the ellipse as $\Lambda=R_{1} / R_{2}$. When $R_{1}$ and $R_{2}$ are identical, the ellipse reduces to a circle.

\section{A. Single impermeable plate}

Fig. 4 displays the free-surface pattern of the diffracted wave field, consisting of both incident and scattering waves, by a submerged impermeable circular plate at $k_{0} R=0.7, v / R=0.2$, and $d / R=2.0$. The white circle corresponds to the frame of the circular plate. Due to the diffraction effect by the submerged plate, a large wave amplification up to 4.0 above the circular plate has been achieved.

Figs. 5 and 6 exhibit the wave exciting force on impermeable elliptical plates and the generated free-surface elevation at $(x, y)=(0,0)$ as a function of normalized wavenumber $k_{0} \sqrt{R_{1} R_{2}}$ for different wave headings. The wave exciting force and free-surface elevation are nondimensionalized with respect to $\pi \rho g a R_{1} R_{2}$ and $a$, respectively. Two aspect ratios $\Lambda=2.0$ and $\Lambda=3.0$ are considered in subplots (a) and (b). The nondimensional immersion depth is $v / \sqrt{R_{1} R_{2}}=0.2$ and the water depth is $d / \sqrt{R_{1} R_{2}}=2.0$. Comparison is made with the case of a circular plate of the same area. It is observed from Fig. 5 that the peak value of the wave exciting force acting on an elliptical plate for all wave incidence angles are apparently smaller than that acting on a circular plate of the same area. Moreover, the peak value of the wave exciting force decreases with increasing the aspect ratio. For the free-surface elevation as in Fig. 6, the tendency is pretty much the same.

Figs. 7 and 8 display the colored plots of the modulus of the free-surface elevation $\|\mathcal{E}\| / a$ at $k_{0} \sqrt{R_{1} R_{2}}=0.7$ related to the diffracted wave field by submerged elliptical plates for aspect ratios $\Lambda=2.0$ and $\Lambda=3.0$, respectively. The submergence and water depth are the same as those adopted in Fig. 6. Comparing subplots (a), (b), and (c), 


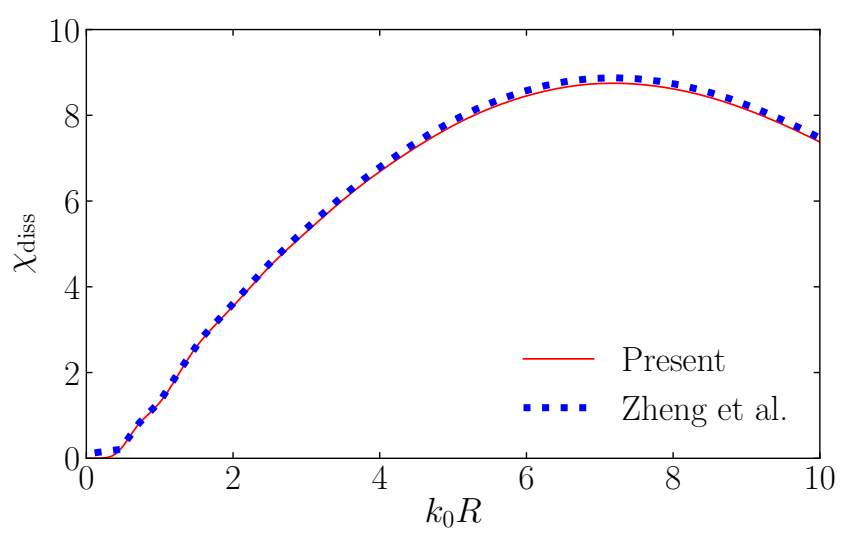

(a)

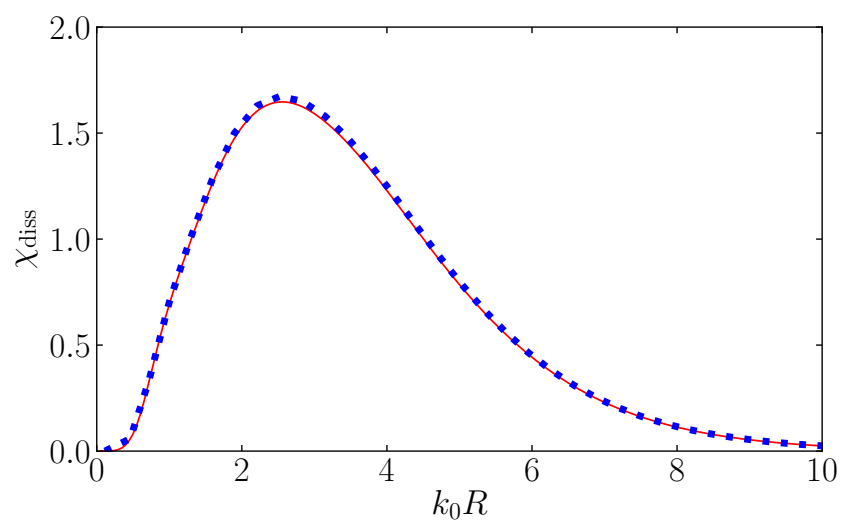

(b)

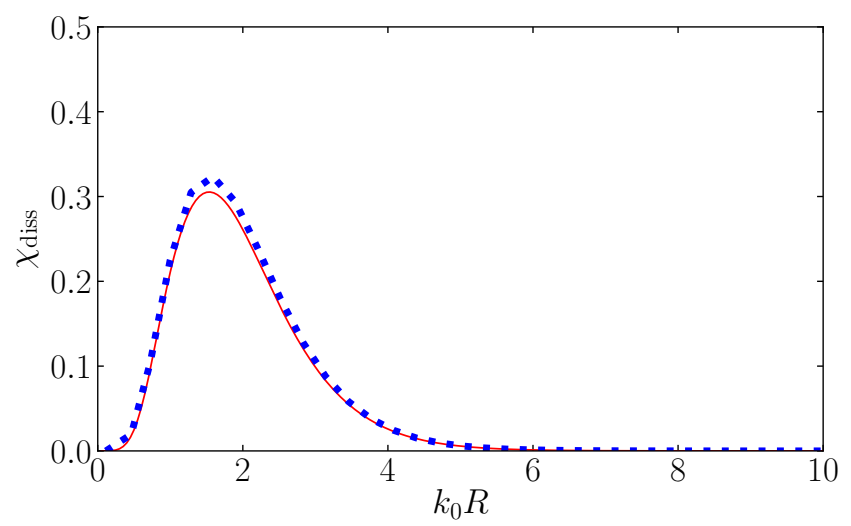

(c)

FIG. 2: Wave energy absorption by a single perforated circular plate for different submerged depths for $d / R=2.0$, and $b=5.0$ : (a) $v / R=0.2$; (b) $v / R=0.5$; (c) $v / R=1.0$. Comparison is made with the analytical solution described in Zheng et al. (2020a).

the maximum wave amplitudes diffracted by the elliptical plates in different wave headings are comparable. In contrast to the free-surface pattern by a circular plate as illustrated in Fig. 4, the maximum wave amplitude is smaller. Moreover, the maximum wave amplitude is de-

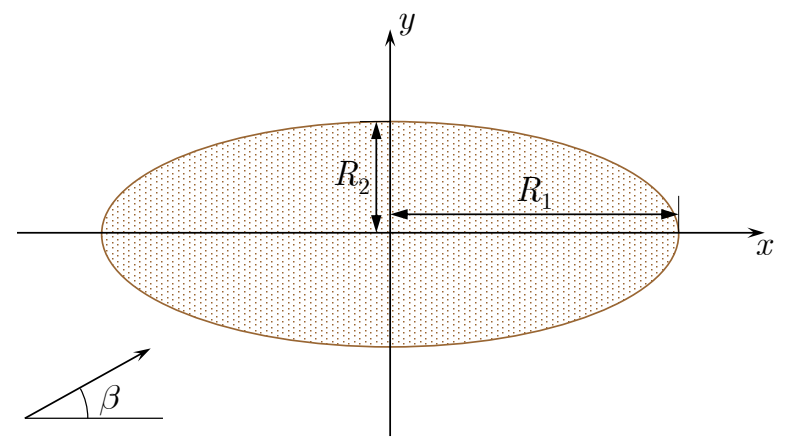

FIG. 3: Sketch of wave interacting with an elliptical plate.

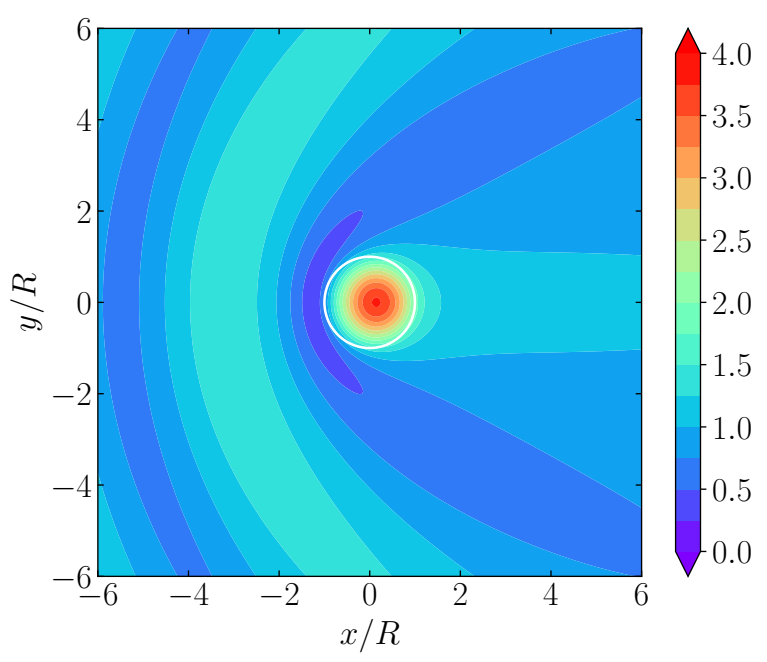

FIG. 4: Colored plot of the modulus of the free-surface elevation $\|\mathcal{E}\| / a$ related to diffracted wave field by a submerged impermeable circular plate for $k_{0} R=0.7$, $v / R=0.2$, and $d / R=2.0$.

creasing with increasing the aspect ratio of the elliptical plate. Therefore, it indicates that the performance of a circular plate is better than that of the elliptical one in terms of wave amplification.

\section{B. Multiple impermeable plates}

As a sequel to the single impermeable plate, wave scattering by multiple plates is now considered. Because the circular plate has better wave amplification performance than the elliptical one of the same area and immersion depth, the focus will be placed on multiple circular plates in this subsection. A deployment of two submerged impermeable circular plates in a side-by-side configuration is first considered. The two circular plates have the identical submergence depth and radius, and the deployment of them is presented in Table $\mathrm{I}$, where $\left(X_{j}, Y_{j}, Z_{j}\right)$ denotes the nondimensional coordinates of the center of the 


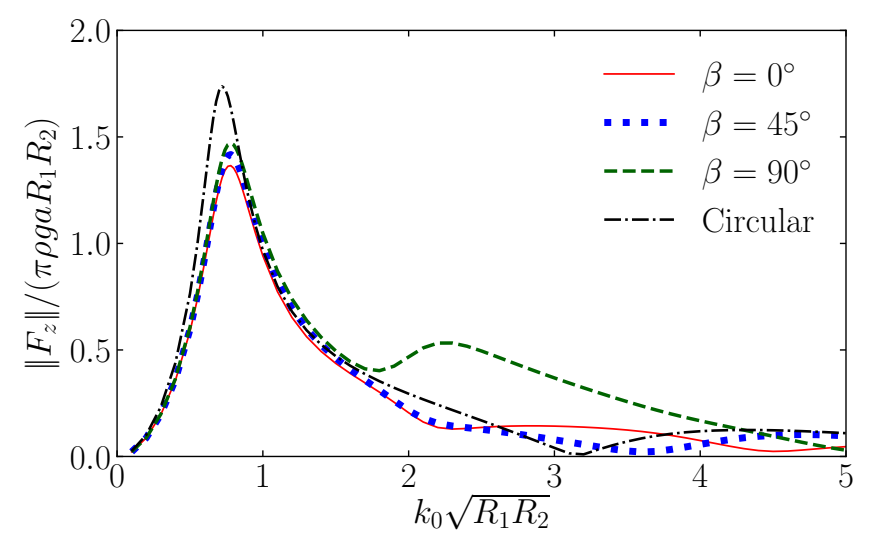

(a)

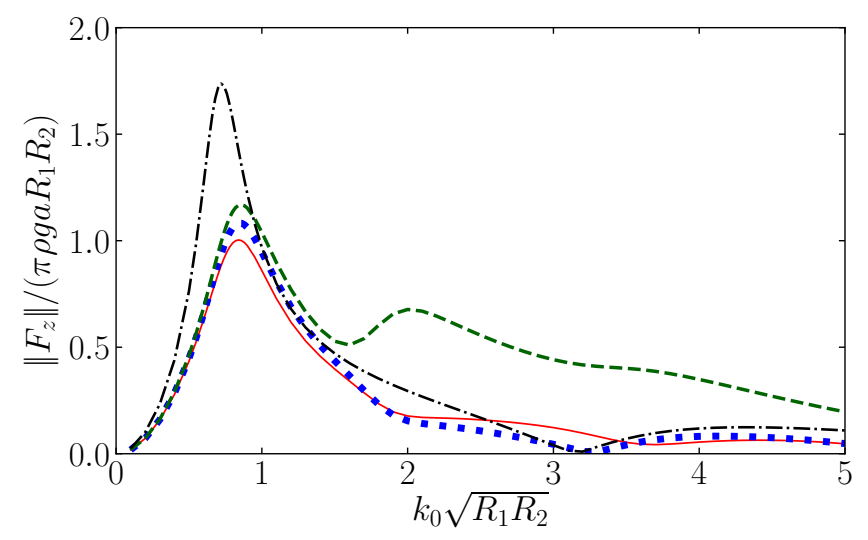

(b)

FIG. 5: Nondimensional wave exciting forces on elliptical plates for different wave headings for $v / \sqrt{R_{1} R_{2}}=0.2$, and $d / \sqrt{R_{1} R_{2}}=2.0$ : (a) $\Lambda=R_{1} / R_{2}=2.0$; (b) $\Lambda=R_{1} / R_{2}=3.0$. Comparison is made with that by a circular plate of the same area.

center of plate $j$, i.e.: $\left(X_{j}, Y_{j}, Z_{j}\right)=\left(x_{j}, y_{j}, z_{j}\right) / \sqrt{R_{1} R_{2}}$.

\begin{tabular}{ccc}
\hline Plate index $j$ & Center's coordinates $\left(X_{j}, Y_{j}, Z_{j}\right)$ \\
1 & $(-1.25$, & $0.00,-0.20)$ \\
2 & $(1.25$, & $0.00,-0.20)$ \\
\hline
\end{tabular}

TABLE I: Coordinates of centers of a two-circular-plate deployment.

Fig. 9 depicts the vertical wave exciting force as a function of the normalized wavenumber $k_{0} R$ for different incident wave directions, including: following sea $\beta=0^{\circ}$, quartering sea $\beta=45^{\circ}$, and beam sea $\beta=90^{\circ}$ displayed in subplots (a), (b), and (c), respectively. The water depth is $d / R=2.0$. In all wave headings, the vertical forces acting on both circular plates reach a peak near $k_{0} R \approx 0.7$. Because of the symmetrical setup, the wave forces experienced by two circular plates under the beam sea excitation are identical as expected. Under the following sea and quartering sea excitations, the peak values

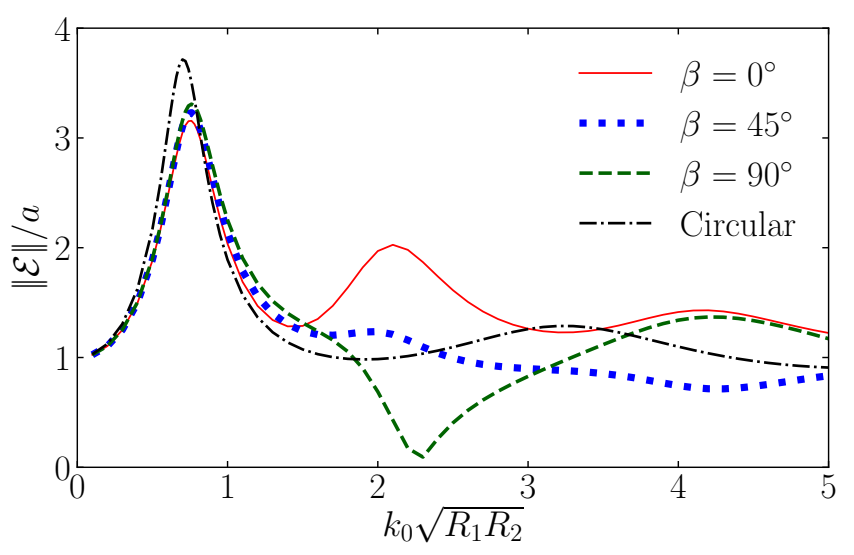

(a)

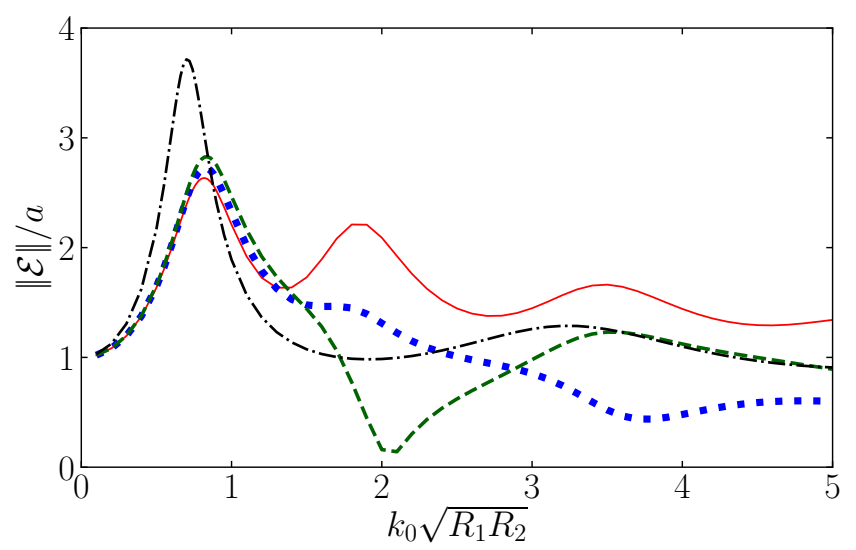

(b)

FIG. 6: Nondimensional free-surface elevations $(x, y)=(0,0)$ induced by submerged elliptical plates for $v / \sqrt{R_{1} R_{2}}=0.2$, and $d / \sqrt{R_{1} R_{2}}=2.0$ for different wave headings: (a) $\Lambda=R_{1} / R_{2}=2.0$; (b) $\Lambda=R_{1} / R_{2}=3.0$. Comparison is made with that by a circular plate of the same area.

are much larger than that in the beam sea condition, and the wave force acting on the weather-side plate is greater than that acting on the lee-side one.

Fig. 10 exhibits the free-surface elevations normalized by the incident wave amplitude for different wave directions. The free-surface elevations at three locations, including: $(x, y) / R=\left(X_{1}, Y_{1}\right),(x, y) / R=(0,0)$, and $(x, y) / R=\left(X_{2}, Y_{2}\right)$, are displayed in subplots (a), (b), and (c), respectively. When $k_{0} R \leq 1.0$, the free-surface elevations above the two circular plates as shown in subplots (a) and (c) are significantly larger than that at the origin displayed in subplot (b). Under the following sea and quartering sea excitations, the free-surface elevations above the centers of the two circular plates reach the maximum near $k_{0} R \approx 0.7$ as shown in the top and bottom panels. Moreover, the free-surface elevation above the weather-side plate is higher than that above the leeside one. 


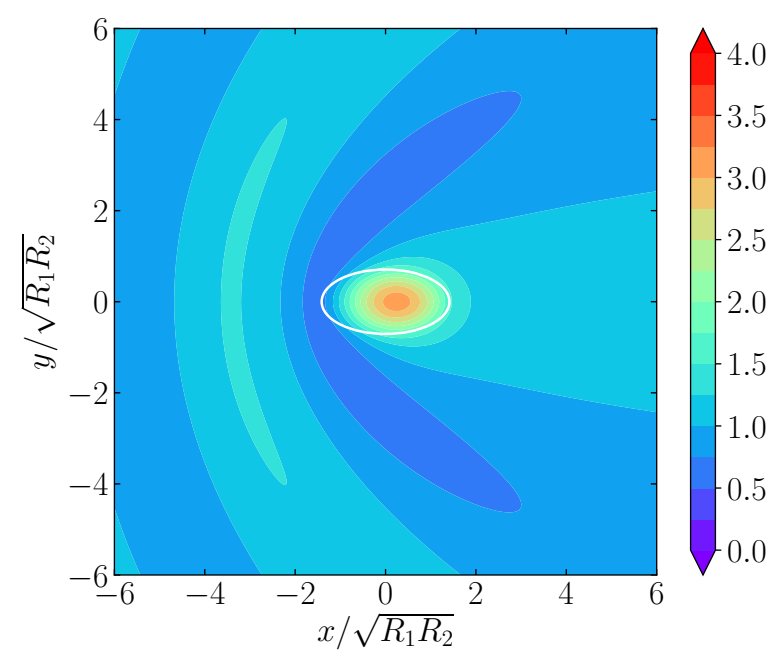

(a)

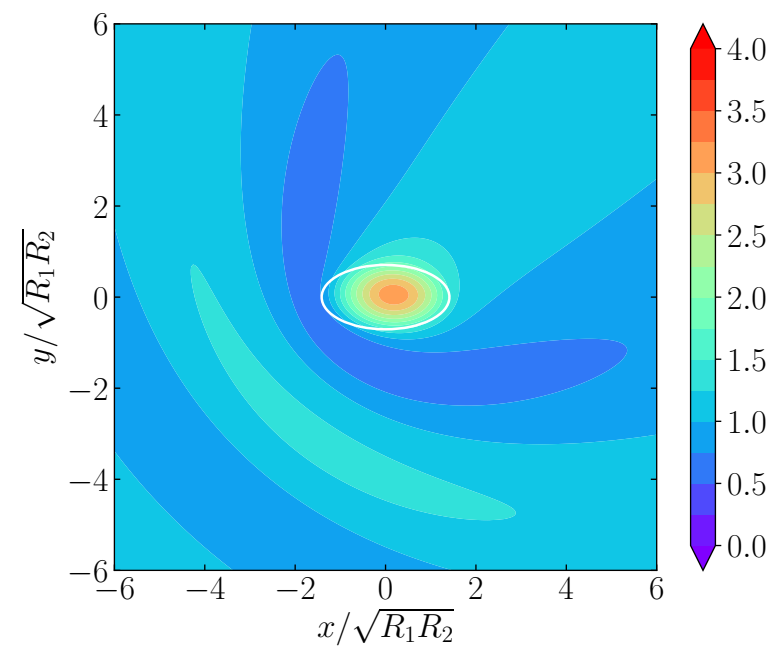

(b)

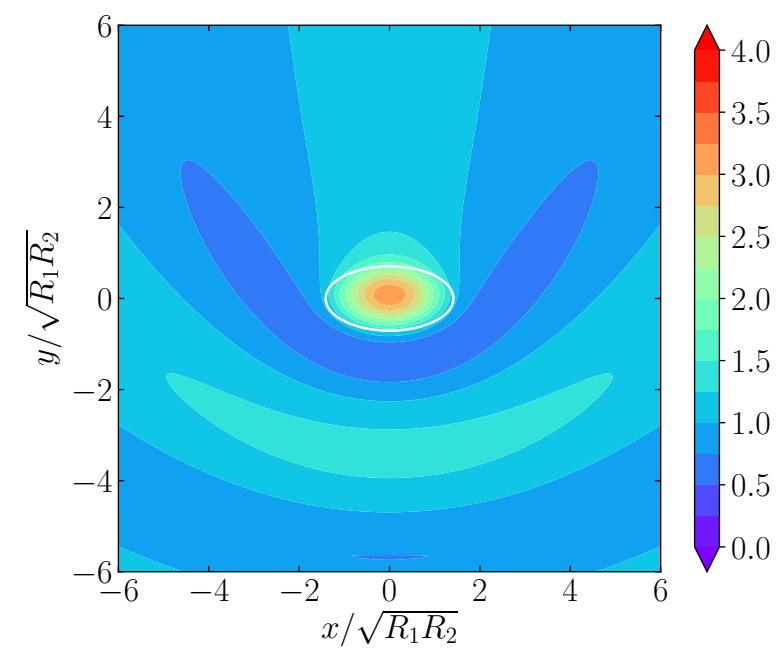

(c)

FIG. 7: Colored plots of the modulus of the free-surface elevation $\|\mathcal{E}\| / a$ related to diffracted waves by a submerged elliptical plate of the aspect ratio

$\Lambda=R_{1} / R_{2}=2.0$ for $k_{0} \sqrt{R_{1} R_{2}}=0.7, v / \sqrt{R_{1} R_{2}}=0.2$, and $d / \sqrt{R_{1} R_{2}}=2.0$ : (a) $\beta=0^{\circ}$; (b) $\beta=45^{\circ}$; (c)

$$
\beta=90^{\circ} \text {. }
$$

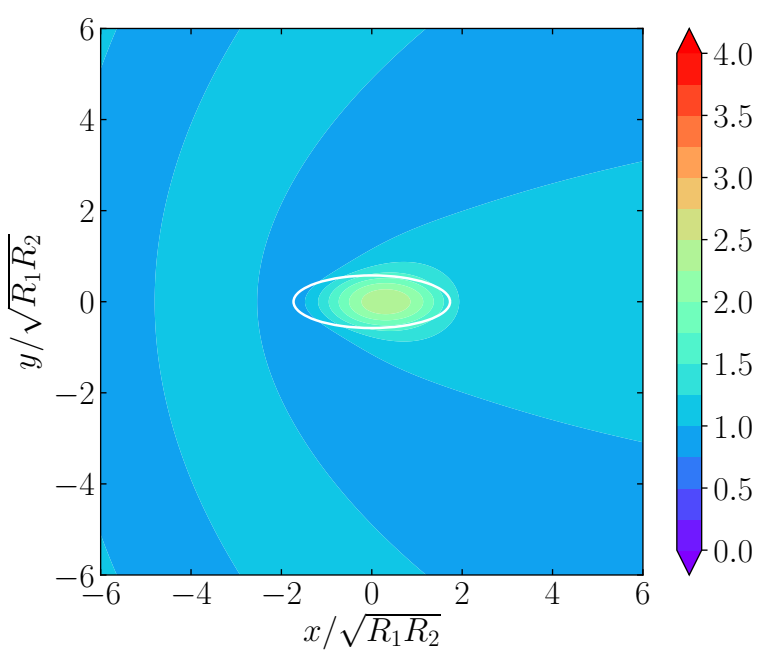

(a)

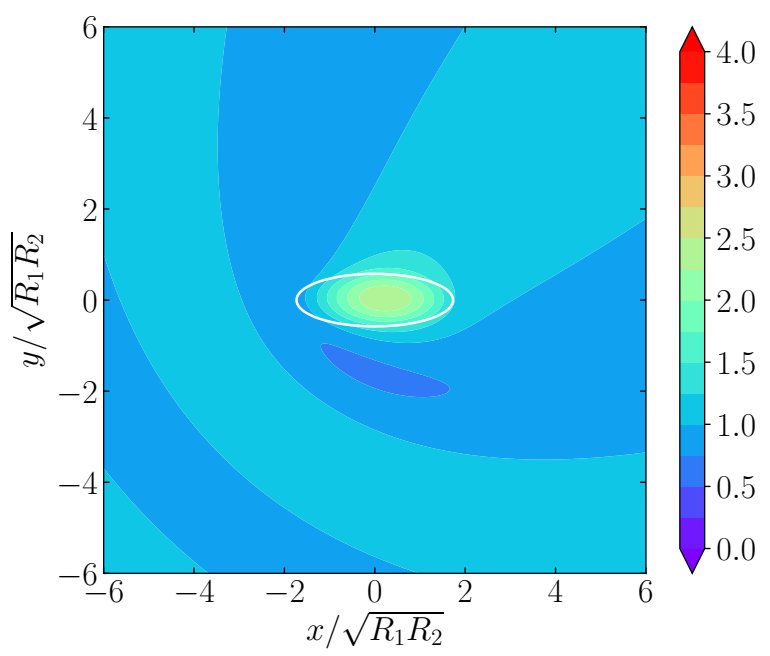

(b)

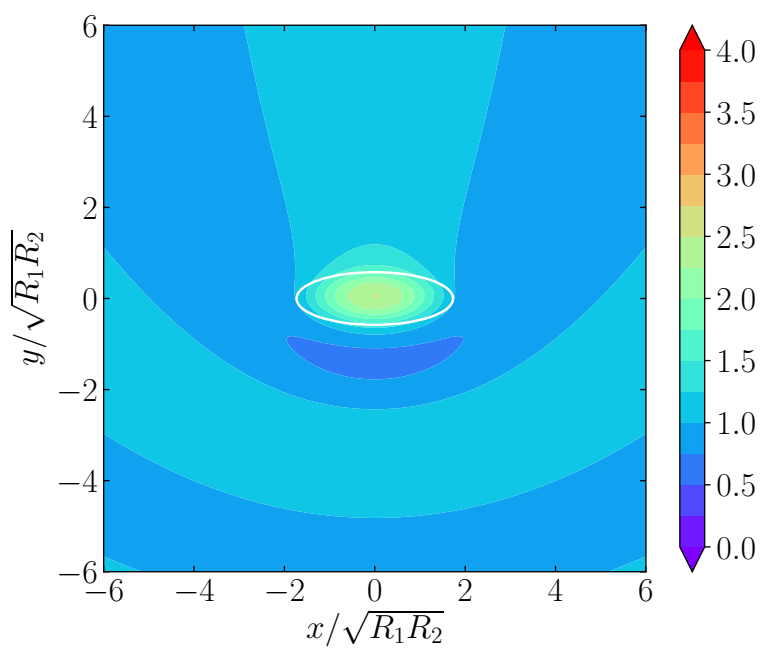

(c)

FIG. 8: Colored plots of the modulus of the free-surface elevation $\|\mathcal{E}\| / a$ related to diffracted waves by a submerged elliptical plate of the aspect ratio

$\Lambda=R_{1} / R_{2}=3.0$ for $k_{0} \sqrt{R_{1} R_{2}}=0.7, v / \sqrt{R_{1} R_{2}}=0.2$, and $d / \sqrt{R_{1} R_{2}}=2.0$ : (a) $\beta=0^{\circ}$; (b) $\beta=45^{\circ}$; (c)

$$
\beta=90^{\circ} \text {. }
$$




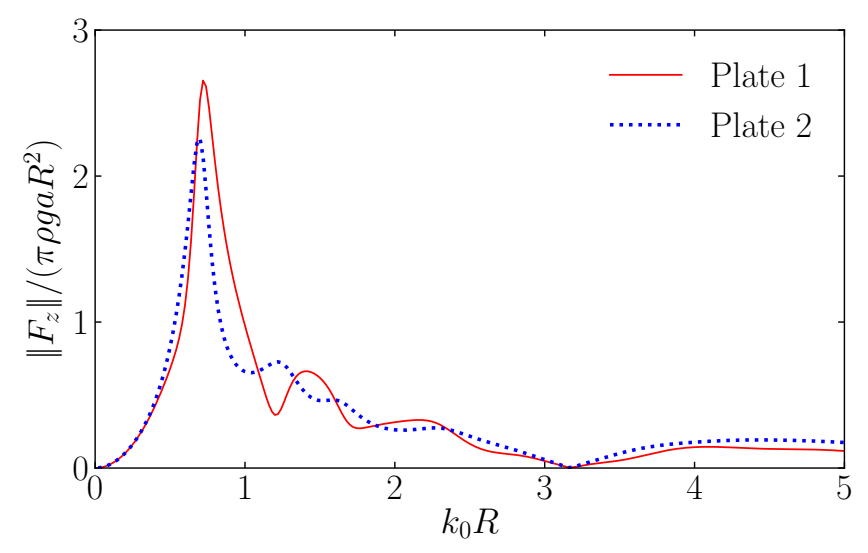

(a)

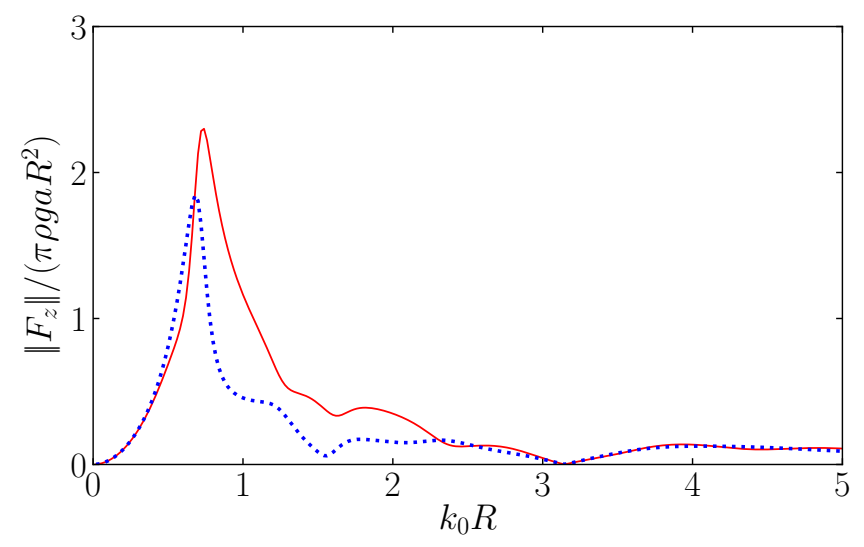

(b)

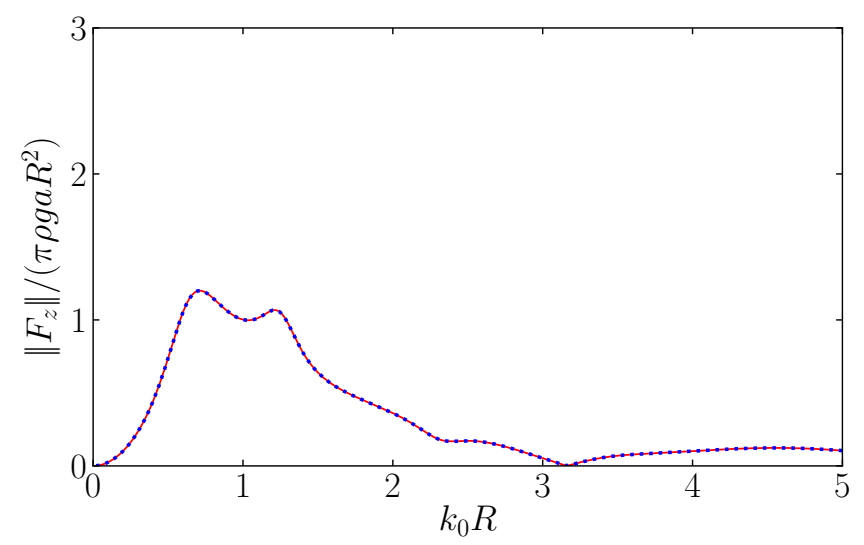

(c)

FIG. 9: Nondimensional vertical wave exciting forces on two impermeable circular plates for different wave headings: (a) $\beta=0^{\circ}$; (b) $\beta=45^{\circ}$; (c) $\beta=90^{\circ}$.

In Fig. 11, the colored plots of the normalized modulus of the free-surface elevations $\|\mathcal{E}\| / a$ for wave diffraction by two impermeable circular plates at $k_{0} R=0.7$ and $d / R=2.0$ are illustrated. The free-surface patterns in following sea $\beta=0^{\circ}$, quarter sea $\beta=45^{\circ}$, and beam sea $\beta=90^{\circ}$ conditions are displayed in subplots (a), (b),

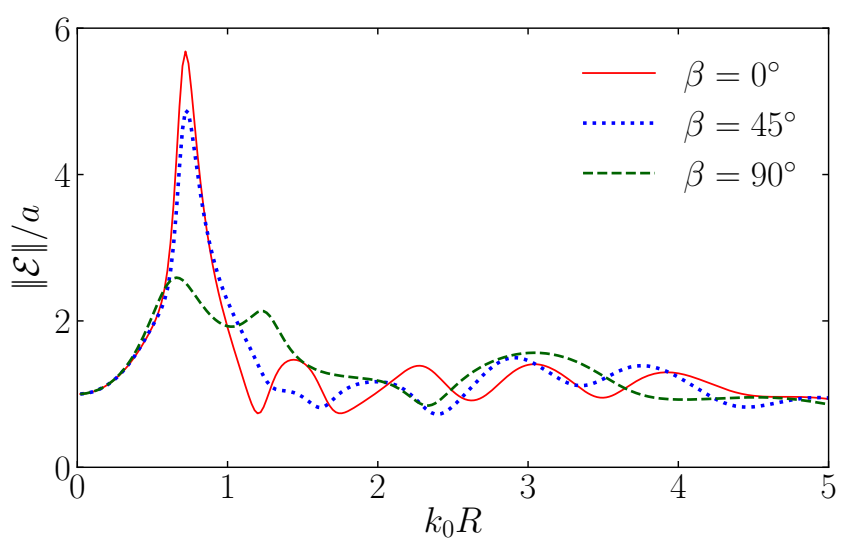

(a)

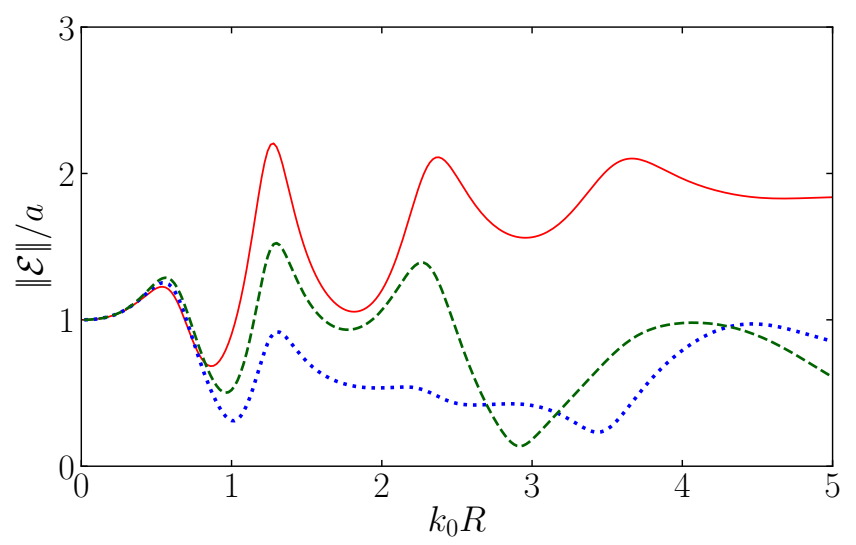

(b)

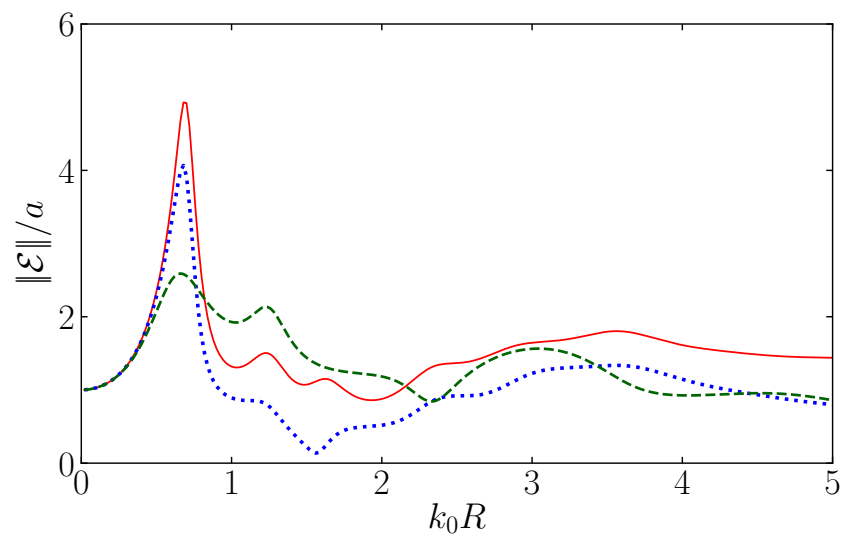

(c)

FIG. 10: Nondimensional free-surface elevation at different locations for a two-circular-plate deployment:

(a) $(x, y)=(-1.25 R, 0)$; (b) $(x, y)=(0,0) ;($ c $)$

$$
(x, y)=(1.25 R, 0) \text {. }
$$

and (c), respectively. In the following sea condition, the wave amplification above the weather-side plate is higher than the lee-side one, and it is much larger than the single plate scenario as shown in Fig. 4. By increasing the 


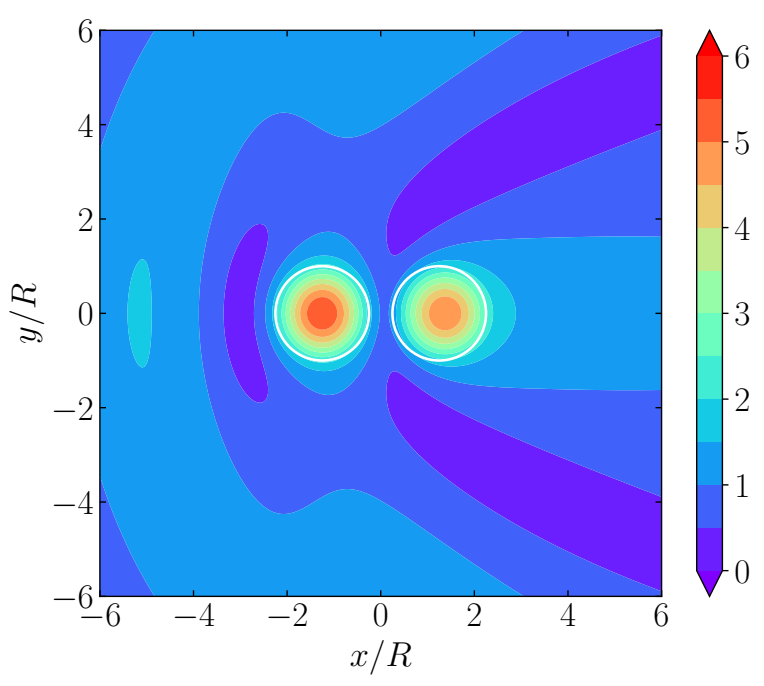

(a)

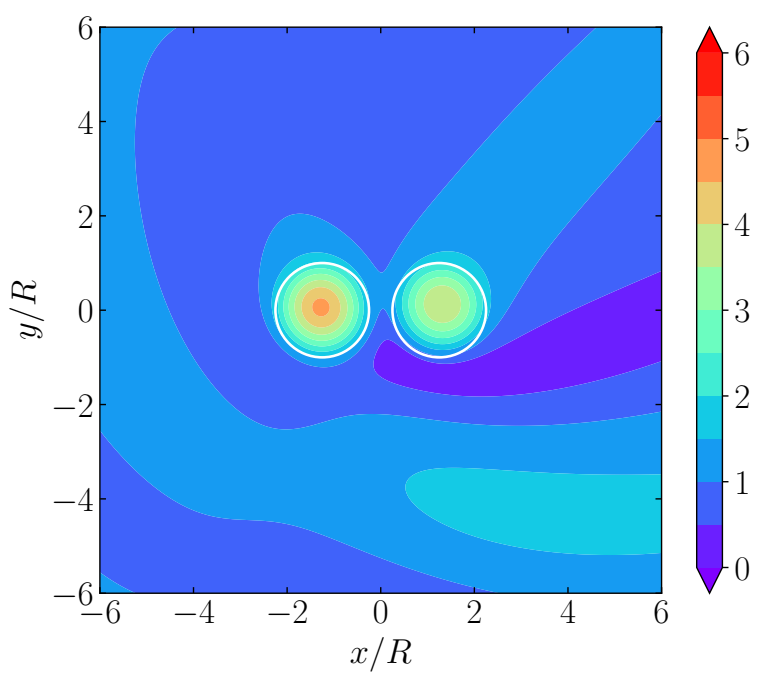

(b)

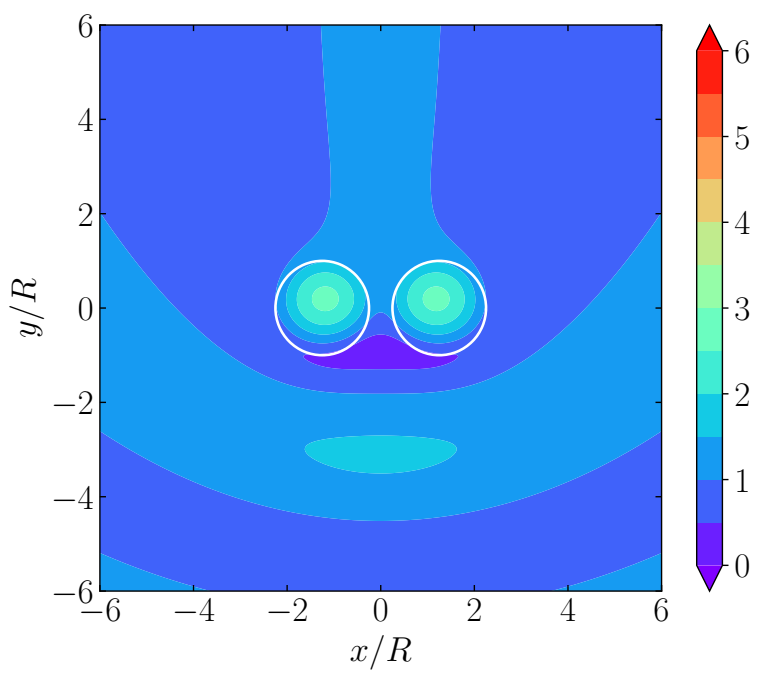

(c)

FIG. 11: Colored plots of the modulus of the free-surface elevation $\|\mathcal{E}\| / a$ related to diffracted waves by two impermeable circular plates at $k_{0} R=0.7$ : (a) $\beta=0^{\circ}$; (b) $\beta=45^{\circ}$; (c) $\beta=90^{\circ}$. wave incidence angle, the maximum wave amplitude is decreasing. The maximum wave amplitude in the beam sea condition as shown in subplot (c) is much smaller than that under following sea and quartering sea excitations as well as the single plate scenario. Therefore, large wave amplification by an array of impermeable circular plates can be achieved if the wave propagates in line with the deployment line. It is expected that the large wave amplification by an array of circular plates can be favorable in enhancing wave power absorption of WECs floating at the free surface (Wang and Zhang, 2021).

Then, wave diffraction by four identical impermeable circular plates is considered, and the deployment of four circular plates is presented in Table II. To avoid overlapping, circular plates ' 3 ' and ' 4 ' have deeper immersions.

\begin{tabular}{cc}
\hline Plate index $j$ & Center's coordinates $\left(X_{j}, Y_{j}, Z_{j}\right)$ \\
1 & $(-1.25,0.00,-0.20)$ \\
2 & $(1.25,0.00,-0.20)$ \\
3 & $(0.00,-1.25,-0.22)$ \\
4 & $(0.00,1.25,-0.22)$ \\
\hline
\end{tabular}

TABLE II: Coordinates of centers of a four-circular-plate deployment.

Fig. 12 depicts the vertical wave exciting forces acting on the four impermeable circular plates as deployed in Table II, and different wave incidence angles, including: following sea $\beta=0^{\circ}$, quartering sea $\beta=45^{\circ}$, and beam sea $\beta=90^{\circ}$, are presented in subplots (a), (b), and (c), respectively. In the present deployment, the lee-side circular plate experiences larger wave force for $k_{0} R \leq 1.0$, which is broader than the range of $k_{0} R$, i.e., $k_{0} R<0.7$, for the two-circular-plate deployment case showing a larger wave force acting on the lee-side circular plate (see Fig. 9). Besides, the oscillation in wave forces is strong indicating that the interference effect is significant.

In Fig. 13, nondimensional free-surface elevations at five locations, including: $(x, y) / R=(0.0,0.0)$, $(-1.25,0.0),(1.25,0.0),(0.0,-1.25)$, and $(0.0,1.25)$, are presented in subplots (a), (b), (c), (d), and (e), respectively. At the origin $(0.0,0.0)$, the free-surface elevation is almost independent of wave headings for $k_{0} R \leq 0.8$ corresponding to long wavelength. At locations above the four circular plates, however, the oscillation in the free-surface elevation is strong due to interference effects. Under the following sea and beam sea excitations, there are two peaks with commensurate peak values above the lee-side plate as shown in the third and bottom panels. Moreover, the peak value above the lee-side plate is larger than that above the weather-side one.

Fig. 14 exhibits the colored plots of the modulus of the normalized free-surface elevations $\|\mathcal{E}\| / a$ related to waves diffracted by a four-circular-plate deployment as in Table II at $k_{0} R=0.7$. White circles correspond to frames of two circular plates of shallower immersion, whereas gray circles to those of deeper submergence. The presence of horizontal overlapping regions indicates that the method 


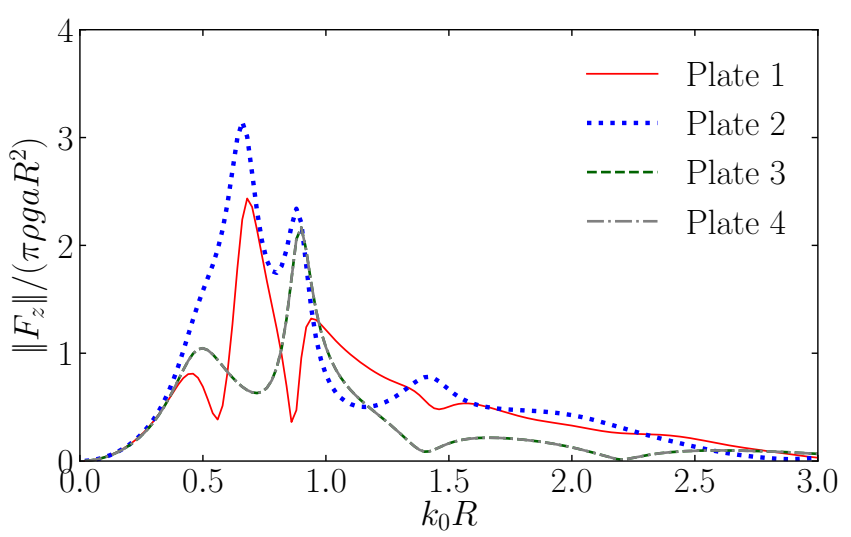

(a)

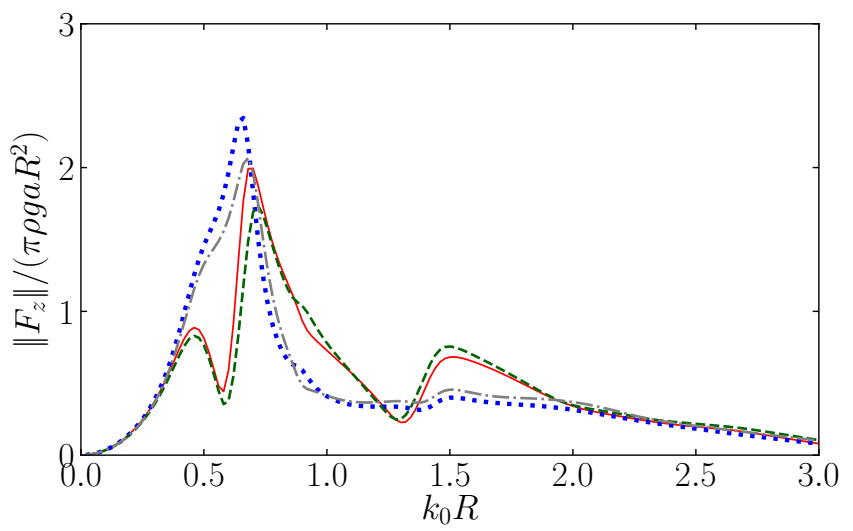

(b)

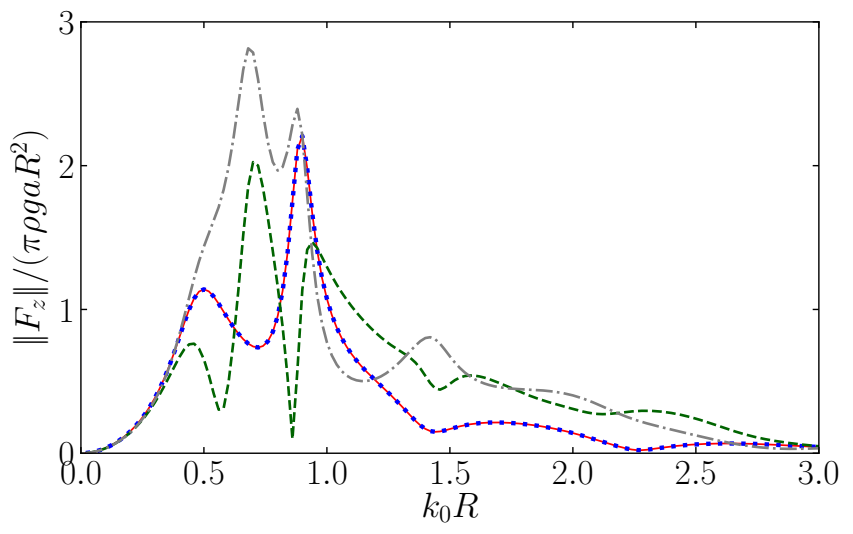

(c)

FIG. 12: Nondimensional vertical wave exciting forces on four impermeable circular plates for different wave headings: (a) $\beta=0^{\circ}$; (b) $\beta=45^{\circ}$; (c) $\beta=90^{\circ}$.

of eigen-function expansion cannot be applied. The freesurface patterns under following sea $\beta=0^{\circ}$, quarter sea $\beta=45^{\circ}$, and beam sea $\beta=90^{\circ}$ excitations are presented in subplots (a), (b), and (c), respectively. In the following sea and beam sea conditions as plotted in subpolots (a) and (c), the free-surface elevations above two circular

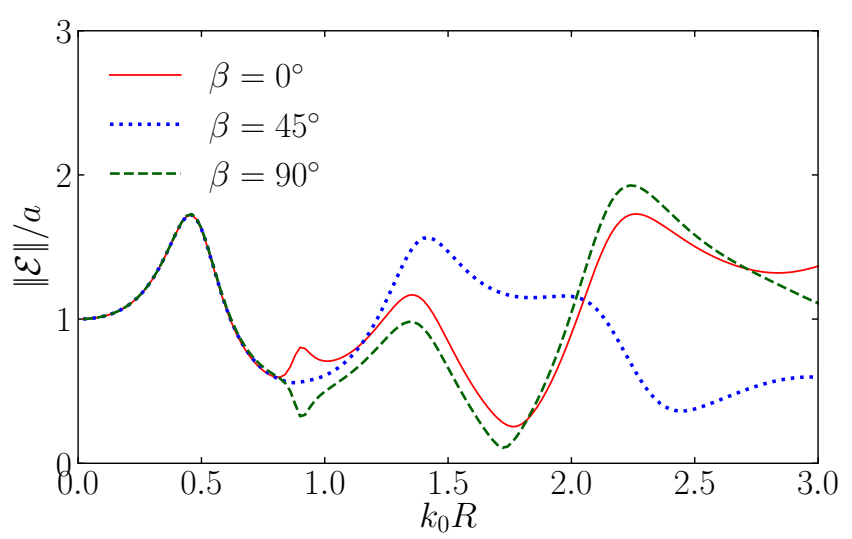

(a)

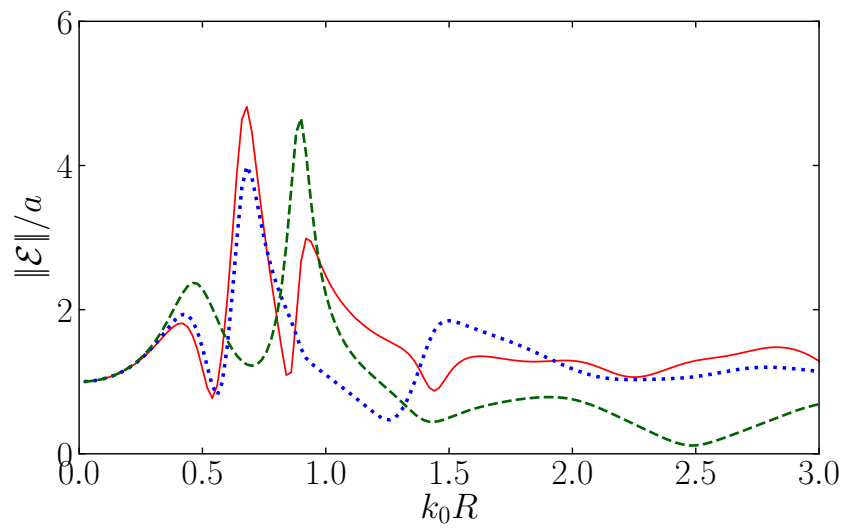

(b)

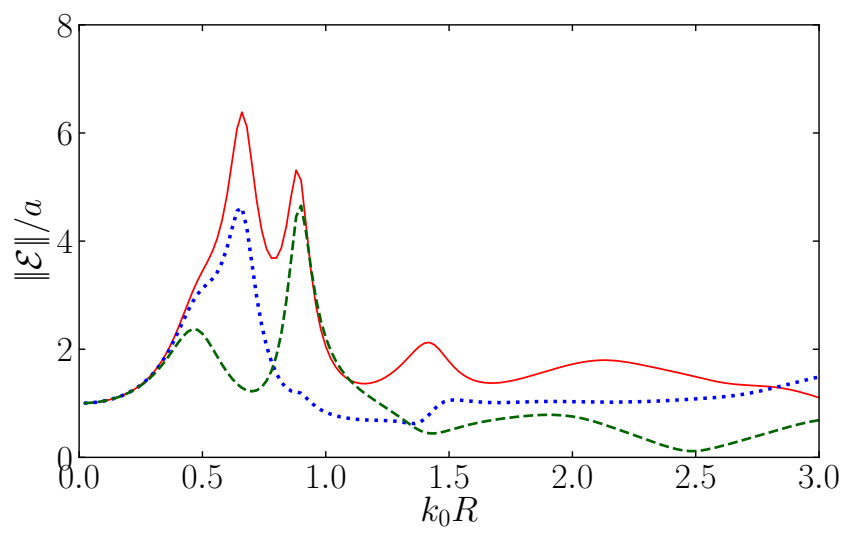

(c)

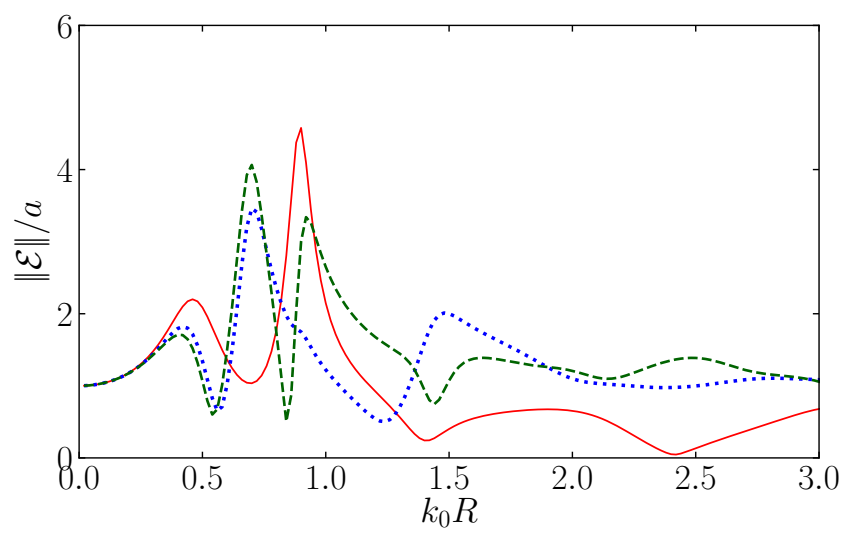

(d) 


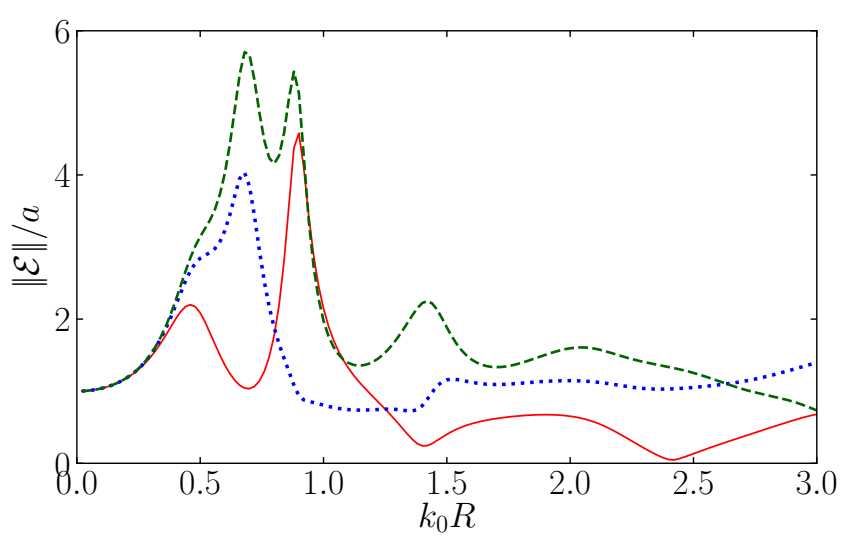

(e)

FIG. 13: Nondimensional free-surface elevation at different locations: (a) $(x, y)=(0,0) ;(\mathrm{b})$

$$
(x, y)=(-1.25 R, 0) ;(\mathrm{c})(x, y)=(1.25 R, 0) ;(\mathrm{d})
$$$$
(x, y)=(0,-1.25 R) ;(\mathrm{e})(x, y)=(0,1.25 R) \text {. }
$$

plates of lateral with respect to the wave direction are small. Apparent wave amplifications above the front and rear circular plates are observed, and larger wave amplification occurs above the rear circular plate. Under the quartering sea excitation as in subplot (b), the freesurface elevations above the circular plate area are larger than elsewhere in general, and wave amplifications over the four circular plates are comparable.

\section{PERFORATED PLATES}

Then, our attention is paid to perforated plates, which are widely adopted to capture wave energy. The energy absorption performances of both circular and elliptical plates will be considered in this section.

\section{A. Single perforated plate}

Wave power absorption by a single perforated plate is first of all considered. Fig. 15 depicts the nondimensional wave power absorption $\chi_{\text {diss }}$ defined in Eq. (14) as a function of normalized wavenumber $k_{0} \sqrt{R_{1} R_{2}}$ for different wave incidence angles, and comparison is made with the wave energy captured by a perforated circular plate of the same area. Aspect ratios $\Lambda=R_{1} / R_{2}=2.0$ and $\Lambda=3.0$ are considered as in subplots (a) and (b), respectively. When the incident wave propagates along the major axis of the ellipse $\left(\beta=0^{\circ}\right)$, the captured wave power is lower than that in quartering sea $\left(\beta=45^{\circ}\right)$ and beam sea $\beta=90^{\circ}$ conditions in the range $3<k_{0} \sqrt{R_{1} R_{2}}<10$ where wave energy absorption ratio is high. Under the quartering sea excitation, the wave energy absorption by an elliptical plate is comparable with the circular one. As the wave incidence direction is in line with the mi-

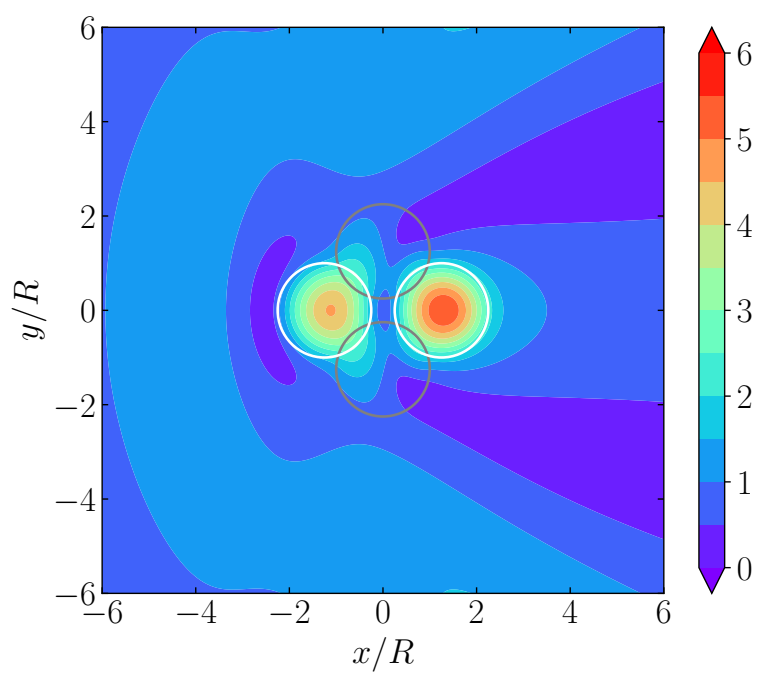

(a)

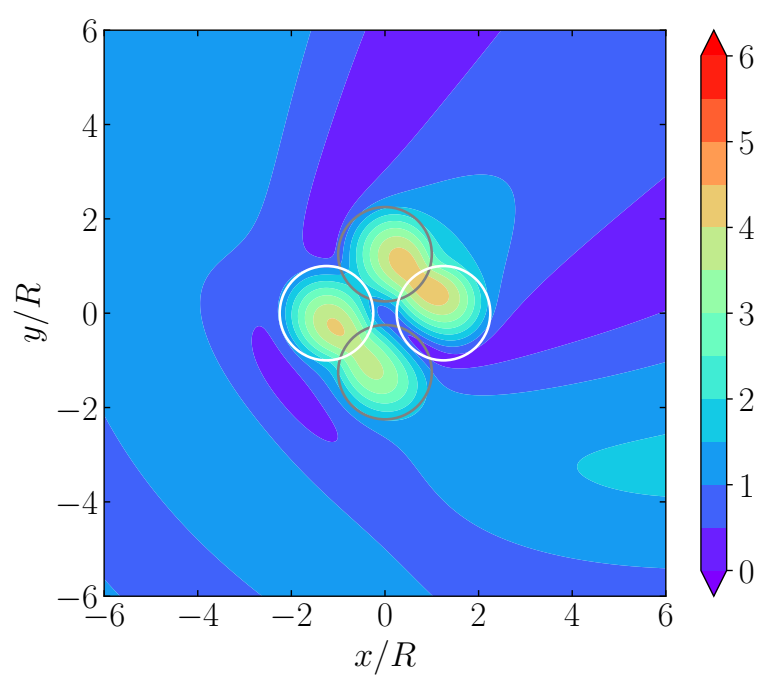

(b)

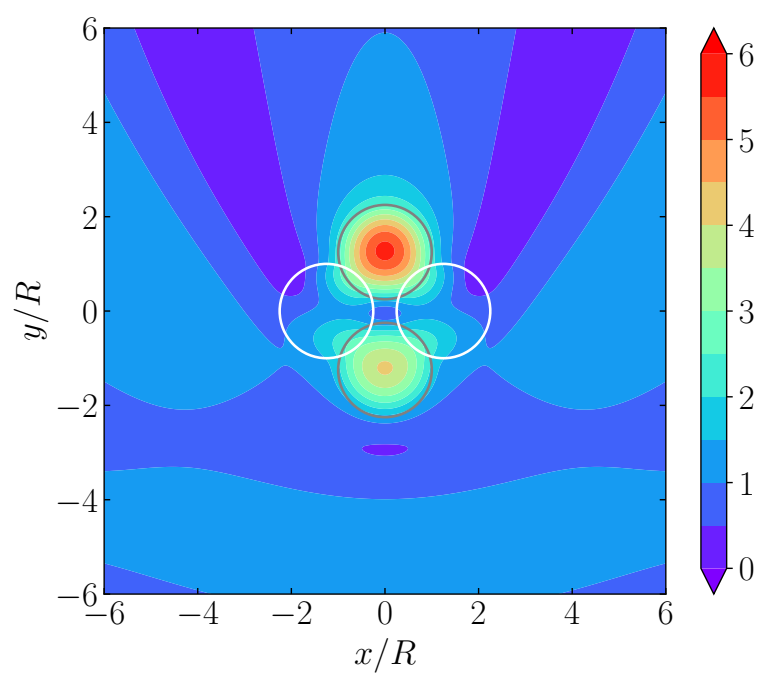

(c)

FIG. 14: Colored plots of the modulus of the free-surface elevation $\|\mathcal{E}\| /$ a related to diffracted waves by four impermeable circular plates at $k_{0} R=0.7$ : (a) $\beta=0^{\circ}$; (b) $\beta=45^{\circ}$; (c) $\beta=90^{\circ}$. 
nor axis of the ellipse $\left(\beta=90^{\circ}\right)$, the performance of the wave power absorption is the best as $3<k_{0} \sqrt{R_{1} R_{2}}<10$, and it is appreciably larger than the wave power by the circular plate of the same area.

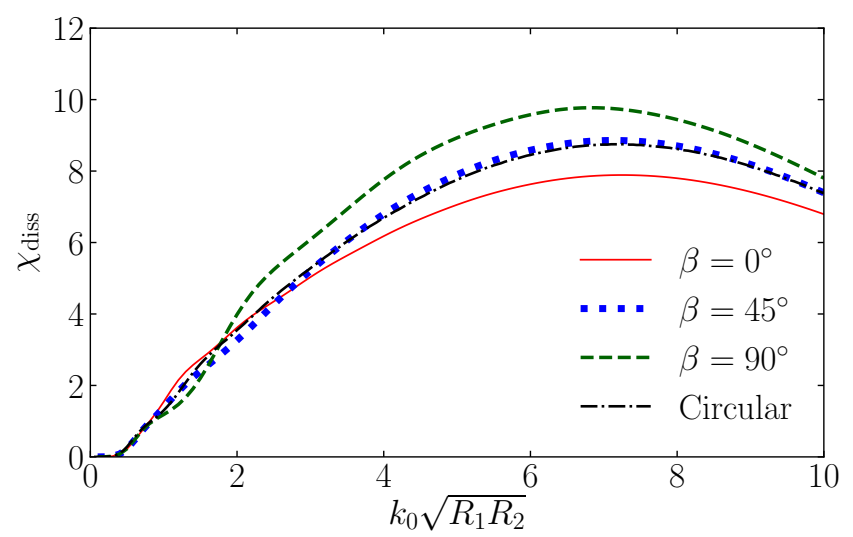

(a)

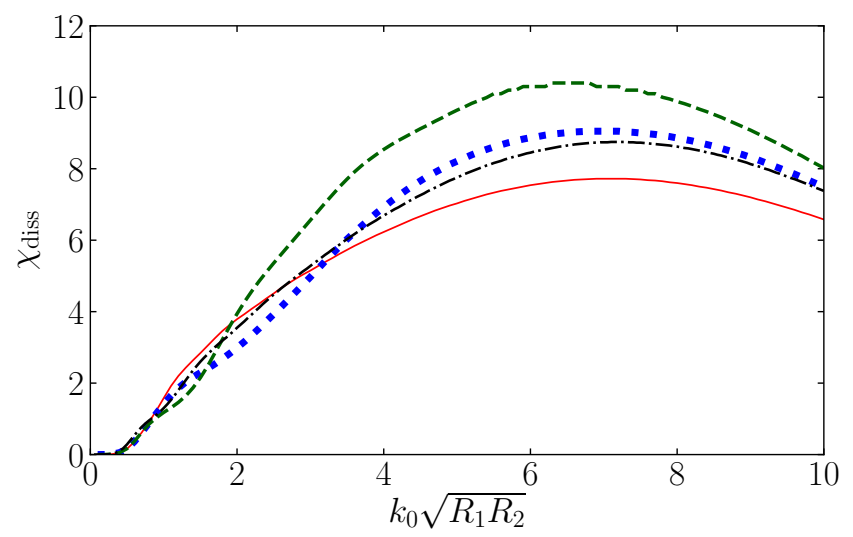

(b)

FIG. 15: Wave power absorption by perforated elliptical plates for different wave headings at $v / \sqrt{R_{1} R_{2}}=0.2$, $d / \sqrt{R_{1} R_{2}}=2.0$ and $b=5.0$ : (a) $\Lambda=R_{1} / R_{2}=2.0$; (b) $\Lambda=R_{1} / R_{2}=3.0$. Comparison is made with the results associated with a perforated circular plate of the same area.

To further investigate the influences of the normalized wavenumber and wave heading on wave power absorption $\chi_{\text {diss }}$, colored plots of wave energy absorption by a single perforated elliptical plate are exhibited in Fig. 16, and results associated with $\Lambda=R_{1} / R_{2}=2.0$ and $\Lambda=3.0$ are presented in subplots (a) and (b), respectively. Wave incidence angles $\beta=0^{\circ}$ and $\beta=90^{\circ}$ correspond to wave propagating along the major and minor axes of the ellipses, respectively. In general, for both the considered $\Lambda$ values, large wave power absorption can be obtained within the range $50^{\circ}<\beta<90^{\circ}$ and $5.0<k_{0} \sqrt{R_{1} R_{2}}<9.0$. In this range, the harnessed wave energy is increasing with the wave incidence angle $\beta$, and a maximum is reached at $\beta=90^{\circ}$. Moreover, by comparing subplots (a) and (b), the elliptical plate of the aspect ratio $\Lambda=3.0$ produces higher energy than that of $\Lambda=2.0$, which indicates a larger aspect ratio is beneficial to wave power absorption.

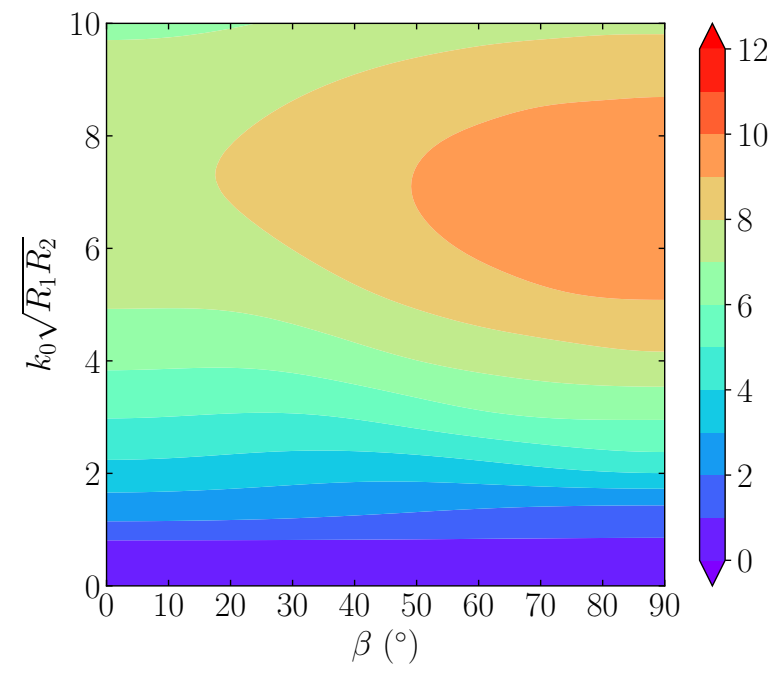

(a)

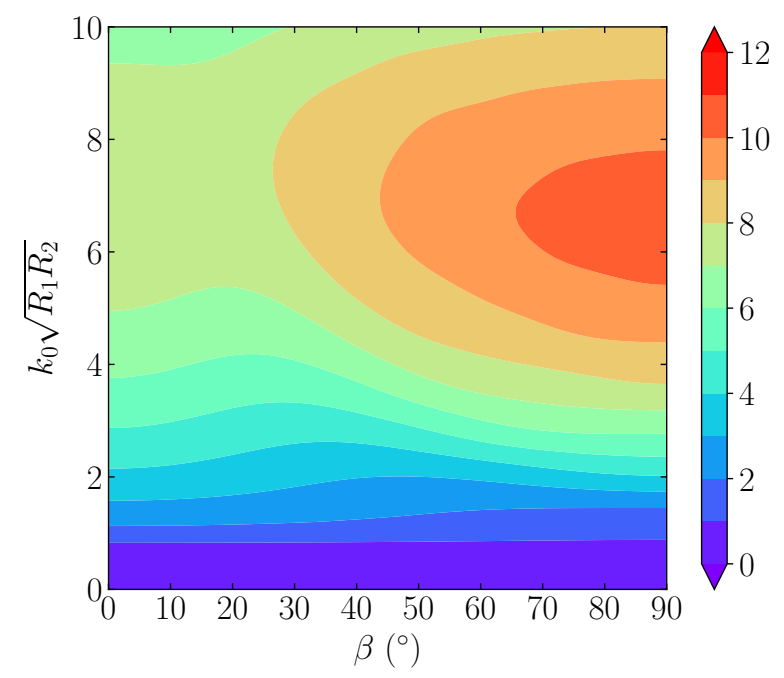

(b)

FIG. 16: Colored plots of the wave energy absorption $\chi_{\text {diss }}$ by a single perforated elliptical plate varying with different normalized wavenumbers $k_{0} \sqrt{R_{1} R_{2}}$ and wave incidence angles $\beta$ at $v / \sqrt{R_{1} R_{2}}=0.2$, and $d / \sqrt{R_{1} R_{2}}=2.0$ : (a) $\Lambda=R_{1} / R_{2}=2.0$; (b) $\Lambda=R_{1} / R_{2}=3.0$.

To delve deeper into the influence of the aspect ratio of ellipse on wave energy harnessing, Fig. 17 displays a colored plot of the wave energy absorption $\chi_{\text {diss }}$ by a single perforated elliptical plate as a function of normalized wavenumbers $k_{0} \sqrt{R_{1} R_{2}}$ and aspect ratios $\Lambda=R_{1} / R_{2}$ in beam sea condition $\left(\beta=90^{\circ}\right)$. This colored plot corroborates the conclusion that the maximum wave power absorption in increasing with the aspect ratio. When the aspect ratio $\Lambda=R_{1} / R_{2}$ is greater than 3.5 , the increment 
in peak value becomes slow, nevertheless the bandwidth of the peak is enlarged.

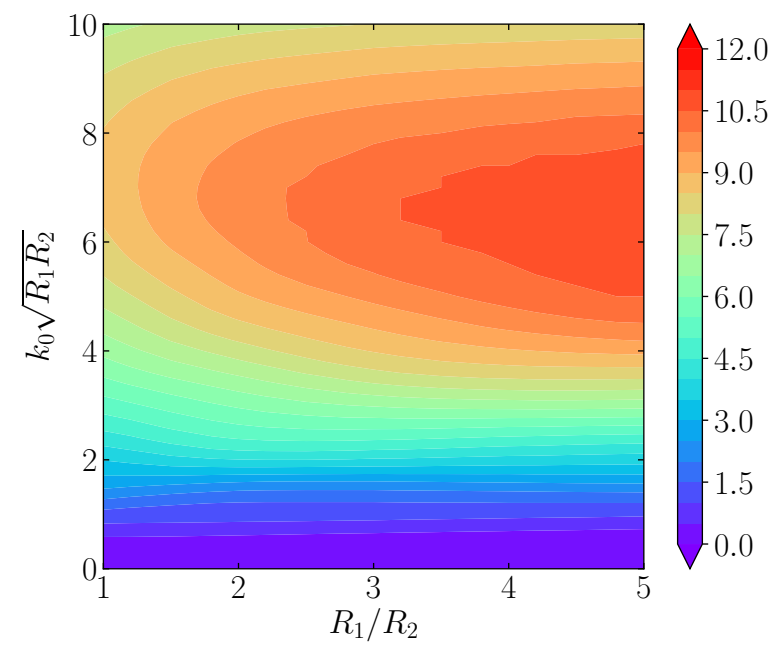

FIG. 17: Colored plot of the wave energy absorption $\chi_{\text {diss }}$ by a single perforated elliptical plate varying with different normalized wavenumbers $k_{0} \sqrt{R_{1} R_{2}}$ and aspect ratios $\Lambda=R_{1} / R_{2}$ at $\beta=90^{\circ}, v / \sqrt{R_{1} R_{2}}=0.2$, and

$$
d / \sqrt{R_{1} R_{2}}=2.0 \text {. }
$$

Fig. 18 illustrates the nondimensional wave energy $\chi_{\text {diss }}$ by a perforated elliptical plate varying with different normalized wavenumbers $k_{0} \sqrt{R_{1} R_{2}}$ and perforation coefficients $b$. The results for aspect ratios $\Lambda=R_{1} / R_{2}=2.0$ and $\Lambda=R_{1} / R_{2}=3.0$ are displayed in subplots (a) and (b) respectively. It can be seen that the maximum wave energy can be achieved by rendering the perforation coefficient $b=4.0$. This plot gives a guidance of the selection of the perforation coefficient.

\section{B. Multiple perforated plates}

Then, we focus on wave scattering by multiple perforated plates in the beam sea condition $\left(\beta=90^{\circ}\right)$ in which the wave power harnessing reaches the maximum. The study presented in Subsection VA indicates that a perforated elliptical plate has better wave energy absorption performance than the circular one. Therefore, only elliptical plates are considered in this subsection, and the aspect ratio is set $\Lambda=R_{1} / R_{2}=3.0$. Fig. 19 shows two typical arrangements of two elliptical plates under the beam sea excitation, including: tandem arrangement, and side-by-side arrangement as illustrated in subplots (a) and (b), respectively.

Table III shows the locations of centers of two identical perforated elliptical plates in a tandem arrangement. The corresponding wave power absorption under the beam sea excitation $\left(\beta=90^{\circ}\right)$ is depicted in Fig. 20, and comparison is made with the results of a single elliptical plate of the same size as in Fig. 15. Due to the symmetrical setup, the wave energy absorption for two

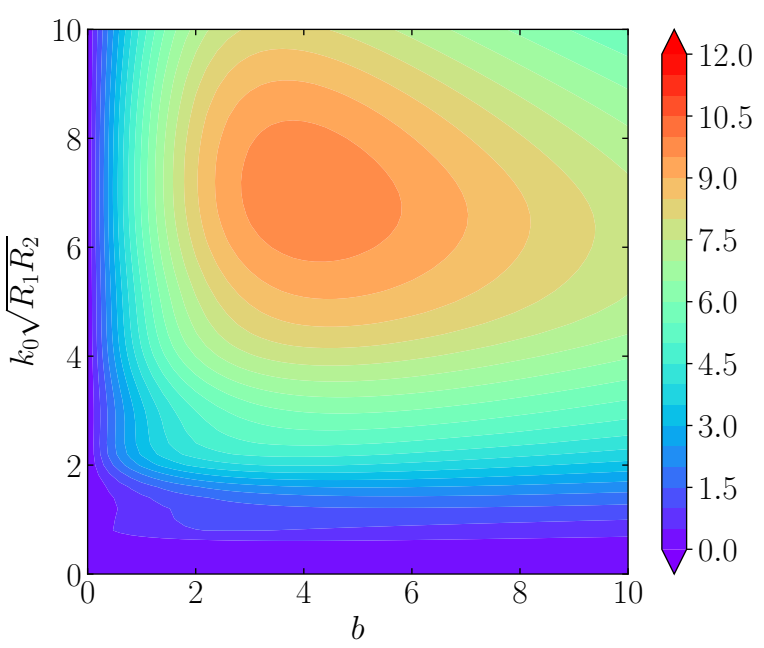

(a)

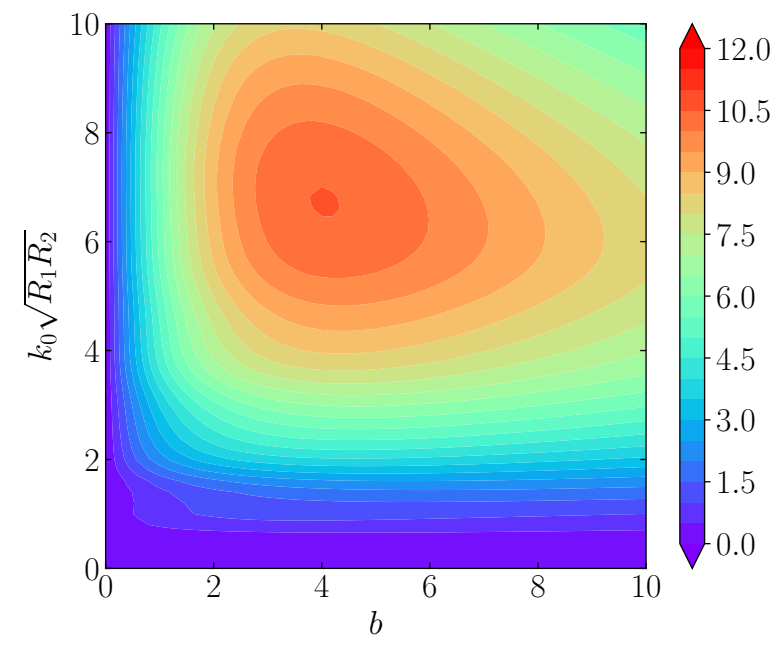

(b)

FIG. 18: Colored plots of the wave energy absorption $\chi_{\text {diss }}$ by a single perforated elliptical plate varying with different normalized wavenumbers $k_{0} \sqrt{R_{1} R_{2}}$ and perforation coefficients $b$ at $v / \sqrt{R_{1} R_{2}}=0.2$, and

$$
\begin{gathered}
d / \sqrt{R_{1} R_{2}}=2.0:\left(\text { a) } \Lambda=R_{1} / R_{2}=2.0 ;\right. \text { (b) } \\
\Lambda=R_{1} / R_{2}=3.0 .
\end{gathered}
$$

plates is identical as expected. Furthermore, although the overall difference from the results of a single elliptical plate is inappreciable, positive interference effect on wave power absorption can be observed for some specified wave conditions, e.g., $k_{0} \sqrt{R_{1} R_{2}}=1.2$ and 2.3 .

\begin{tabular}{cc}
\hline Plate index $j$ Center's coordinates $\left(X_{j}, Y_{j}, Z_{j}\right)$ \\
1 & $(-2.00,0.00,-0.20)$ \\
2 & $(2.00,0.00,-0.20)$ \\
\hline
\end{tabular}

TABLE III: Coordinates of centers of two elliptical plates in a tandem arrangement.

Table IV exhibits the locations of centers of two identi- 


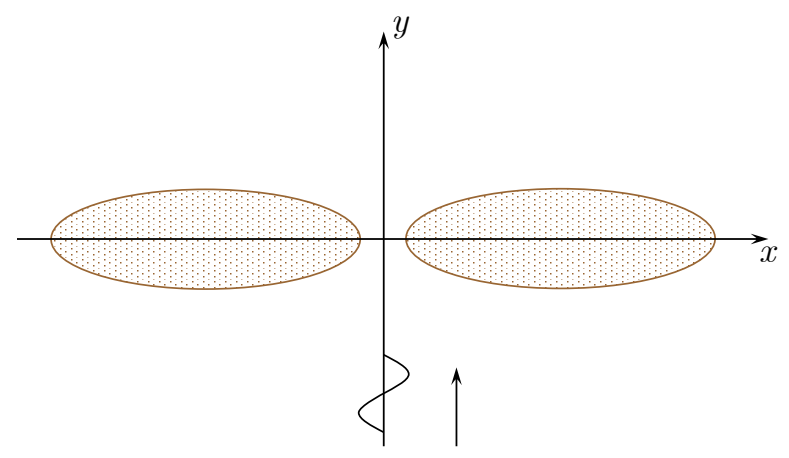

(a)

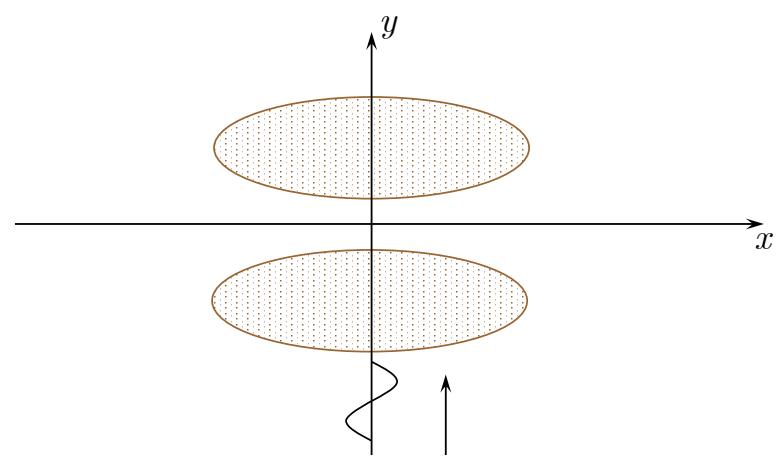

(b)

FIG. 19: Sketch of two deployments of two elliptical plates under the beam sea excitation: (a) Tandem arrangement; (b) Side-by-side arrangement.

cal perforated elliptical plates in a side-by-side arrangement, and the corresponding wave power absorption is depicted in Fig. 21. Again, comparison is made with the results of a single elliptical plate of the same size presented in Fig. 15. In this setup, the wave power absorption by the weather-side plate is consistent with that by a single elliptical plate indicating that the interference is inconsequential. Nevertheless, the wave energy captured by the lee-side plate is reduced significantly resulting in the drop in the averaged power harnessed. The reason is that the weather-side plate acts as a breakwater resulting in considerable wave attenuation downstream, and thus the wave power absorbed by the lee-side plate is reduced dramatically. Therefore, the side-by-side arrangement is not recommended in the deployment of elliptical plates from the perspective of wave power absorption.

\begin{tabular}{cc}
\hline Plate index $j$ Center's coordinates $\left(X_{j}, Y_{j}, Z_{j}\right)$ \\
1 & $(0.00,-1.00,-0.20)$ \\
2 & $(0.00,1.00,-0.20)$ \\
\hline
\end{tabular}

TABLE IV: Coordinates of centers of two elliptical plates in a side-by-side arrangement.

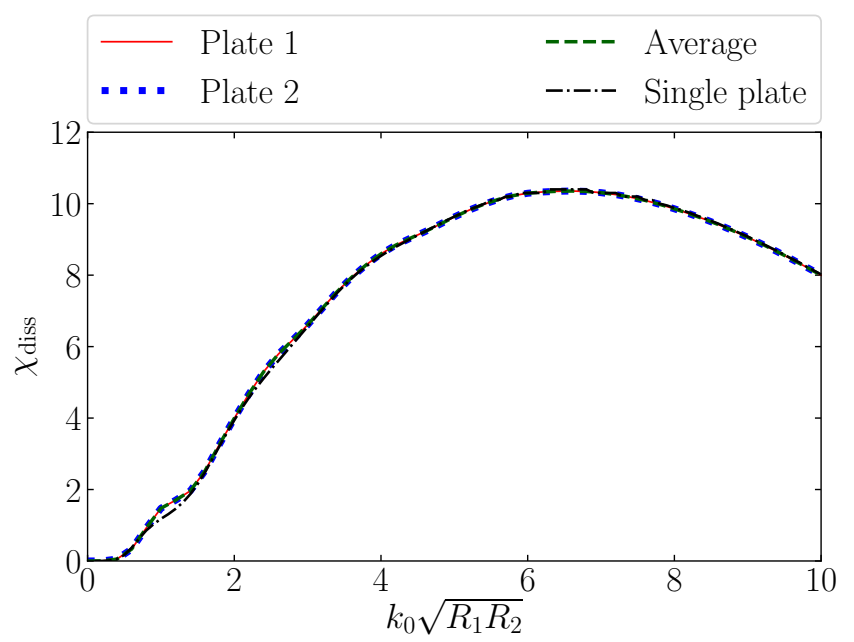

FIG. 20: Wave energy absorption by two perforated elliptical plates in a tandem arrangement for $\beta=90^{\circ}$, $v / \sqrt{R_{1} R_{2}}=0.2$, and $d / \sqrt{R_{1} R_{2}}=2.0$. Comparison is made with the results of a single elliptical plate.

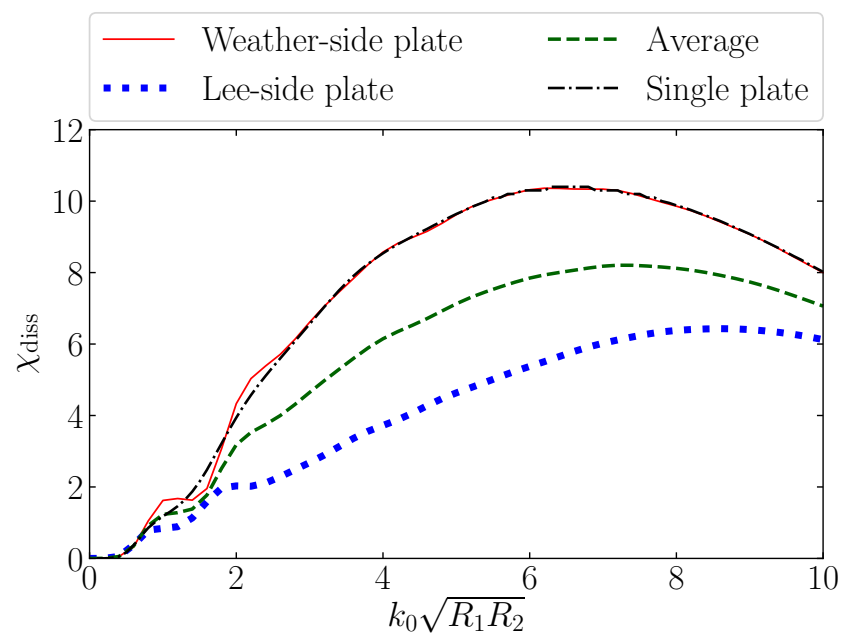

FIG. 21: Wave energy absorption by two perforated elliptical plates in aside-by-side arrangement for $\beta=90^{\circ}, v / \sqrt{R_{1} R_{2}}=0.2$, and $d / \sqrt{R_{1} R_{2}}=2.0$. Comparison is made with the results of a single elliptical plate.

\section{CONCLUSIONS AND FUTURE PERSPECTIVES}

Wave scattering by circular and elliptical plates is investigated by solving the hypersingular integral equation under an assumption of small wave steepness $k_{0} a \ll 1$ with the focus on wave energy utilization. Both impermeable and perforated plates are considered. The former is associated with wave amplification, whereas the latter with wave energy absorption. Through this study, the following conclusions can be drawn:

1. The impermeable plates can be used as a 'wave 
lens' to achieve wave amplification. The circular plate performs better than the elliptical one of the same area, and the wave interference effect due to multiple circular plates can be made use of to further increase the wave amplification.

2. Analogous to the damping effect of the PTO, the perforated plate can be adopted to harness wave energy. Compared to the circular plate, the elliptical plate has better wave power absorption performance provided the incident wave propagates along the minor axis. For an array of elliptical plates, the tandem arrangement with the major axis aligned and deployment line perpendicular to the incident wave direction is recommended.

The present work is focused on the study on water wave interaction with horizontal rigid plates. In spite of this, the model developed in this paper can be employed to solve wave diffraction and radiation by any thin-walled structures, e.g., open cylinder, arc-shaped breakwater, etc. Moreover, the application of hypersingular integral equations to deal with surface-piecing thin-walled structures does not suffer from irregular frequencies associated with the uniqueness and existence of the solution (Liang et al., 2020). The present algorithm can also be extended to account for hydroelasticity so that flexible structures can be dealt with, and this will be reported in another work. The linear approximation for small wave steepness was employed throughout the paper and viscous effects were out ruled; hence the model is not suitable for interactions between extreme waves and structures, which will be left for future work.

\section{ACKNOWLEDGMENT}

This research is supported by $\mathrm{A}^{*} \mathrm{STAR}$ Science and Engineering Research Council, Grant number 17219 00089 under the Marine \& Offshore Strategic Research Programme (M\&O SRP). S. Z. gratefully acknowledges the State Key Laboratory of Hydroscience and Engineering (Tsinghua University) for supporting part of this work through the Open Research Fund Program (grant no. sklhse-2021-E-02).

\section{DATA AVAILABILITY STATEMENTS}

The data that support the findings of this study are available from the corresponding author upon reasonable request.

\section{REFERENCES}

Abramowitz, M. and Stegun, I. A., Handbook of Mathematical Functions: with Formulas, Graphs, and Mathematical Tables, 55 (National Bureau of Standards, 1964).
Behera, H. and Sahoo, T., "Hydroelastic analysis of gravity wave interaction with submerged horizontal flexible porous plate," Journal of Fluids and Structures 54, 643-660 (2015).

Bottom II, R. G., Borazjani, I., Blevins, E. L., and Lauder, G. V., "Hydrodynamics of swimming in stingrays: numerical simulations and the role of the leading-edge vortex," Journal of Fluid Mechanics 788, 407-443 (2016).

Chen, X. B., "Evaluation de la fonction de Green du probleme de diffraction/radiation en profondeur d'eau finie - Une nouvelle méthode rapide et précise," in Actes des 4e Journées de l'Hydrodynamique, Nantes, France (1993) pp. 371-384.

Chen, X. B., Dias, F., and Duan, W. Y., "Introduction of dissipation in potential flows," in Proceeding of the 7th International Workshop on Ship Hydrodynamics, Shanghai, China, edited by D. C. Wan (2011).

Chwang, A. T., "A porous-wavemaker theory," Journal of Fluid Mechanics 132, 395-406 (1983).

Chwang, A. T. and Chan, A. T., "Interaction between porous media and wave motion," Annual Review of Fluid Mechanics 30, 53-84 (1998).

Faltinsen, O. M., Sea Loads on Ships and Offshore Structures (Cambridge University Press, 1993).

Hariri Nokob, M. and Yeung, R. W., "Diffraction and radiation loads on open cylinders of thin and arbitrary shapes," Journal of Fluid Mechanics 772, 649-677 (2015).

Huang, Z., Li, Y., and Liu, Y., "Hydraulic performance and wave loadings of perforated/slotted coastal structures: A review," Ocean Engineering 38, 1031-1053 (2011).

Li, Z. F., Wu, G. X., and Ji, C. Y., "Interaction of wave with a body submerged below an ice sheet with multiple arbitrarily spaced cracks," Physics of Fluids 30, 057107 (2018).

Li, Z. F., Wu, G. X., and Shi, Y. Y., "Wave diffraction by a circular crack in an ice sheet floating on water of finite depth," Physics of Fluids 30, 117103 (2018).

Liang, H., Ouled Housseine, C., Chen, X. B., and Shao, Y., "Efficient methods free of irregular frequencies in wave and solid/porous structure interactions," Journal of Fluids and Structures 98, 103130 (2020).

Liang, H., Shao, Y., and Chen, J., "Higher-order derivatives of the Green function in hyper-singular integral equations," European Journal of Mechanics - B/Fluids 86, 223-230 (2021).

Liu, Y., Cong, P., Gou, Y., Yoshida, S., and Kashiwagi, M., "Enhanced Endo's approach for evaluating free-surface Green's function with application to wave-structure interactions," Ocean Engineering 207, 107377 (2020).

Mackay, E. B. L., "Consistent expressions for the free-surface Green function in finite water depth," Applied Ocean Research 93, 101965 (2019).

Mackay, E. B. L., Liang, H., and Johanning, L., "A BEM model for wave forces on structures with thin porous elements," Journal of Fluids and Structures 102, 103246 (2021).

Martin, P. A. and Farina, L., "Radiation of water waves by a heaving submerged horizontal disc," Journal of Fluid Mechanics 337, 365-379 (1997).

Mei, C. C., Stiassnie, M., and Yue, D. K. P., Theory and applications of ocean surface waves (3rd edition) (World Scientific, 2017).

Meylan, M. H., Bennetts, L. G., Cavaliere, C., Alberello, A., and Toffoli, A., "Experimental and theoretical models of waveinduced flexure of a sea ice floe," Physics of Fluids 27, 041704 (2015).

Meylan, M. H., Bennetts, L. G., and Peter, M. A., "Water-wave scattering and energy dissipation by a floating porous elastic plate in three dimensions," Wave Motion 70, 240-250 (2017).

Molin, B., Hydrodynamique des Structures Offshore (Editions Technip, 2002).

Molin, B., "Hydrodynamic modeling of perforated structures," Applied Ocean Research 33, 1-11 (2011).

Newman, J. N., Marine Hydrodynamics (Massachusetts Institute of Technology Press, 1977). 
Newman, J. N., "Algorithms for the free-surface Green function," Journal of Engineering Mathematics 19, 57-67 (1985).

Newman, J. N., "Amplification of waves by submerged plates," in Proceedings of the 30th international workshop on water waves and floating bodies, Bristol, UK, edited by R. Porter and J. Zang (2015) pp. 153-156.

Parsons, N. F. and Martin, P. A., "Scattering of water waves by submerged plates using hypersingular integral equations," Applied Ocean Research 14, 313-321 (1992).

Porter, R., "Linearised water wave problems involving submerged horizontal plates," Applied Ocean Research 50, 91-109 (2015).

Renzi, E., "Hydroelectromechanical modelling of a piezoelectric wave energy converter," Proceedings of the Royal Society A: Mathematical, Physical and Engineering Sciences 472, 20160715 (2016).

Renzi, E. and Dias, F., "Resonant behaviour of an oscillating wave energy converter in a channel," Journal of Fluid Mechanics 701, 482-510 (2012).

Sarkar, D., Renzi, E., and Dias, F., "Wave farm modelling of oscillating wave surge converters," Proceedings of the Royal Society A: Mathematical, Physical and Engineering Sciences 470,
20140118 (2014).

Selvan, S. A., Gayathri, R., Behera, H., and Meylan, M. H., "Surface wave scattering by multiple flexible fishing cage system," Physics of Fluids 33, 037119 (2021).

Stamnes, J. J., Løvhaugen, O., Spjelkavik, B., Mei, C. C., Lo, E., and Yue, D. K. P., "Nonlinear focusing of surface waves by a lens-theory and experiment," Journal of Fluid Mechanics 135, 71-94 (1983).

Wang, C. and Zhang, Y., "Hydrodynamic performance of an offshore oscillating water column device mounted over an immersed horizontal plate: A numerical study," Energy 222, 119964 (2021).

Wehausen, J. V. and Laitone, E. V., "Surface waves," Hanbuch der Physik 9, 446-778 (1960).

Zheng, S., Meylan, M. H., Greaves, D., and Iglesias, G., "Waterwave interaction with submerged porous elastic disks," Physics of Fluids 32, 047106 (2020a).

Zheng, S., Meylan, M. H., Zhu, G., Greaves, D., and Iglesias, G., "Hydroelastic interaction between water waves and an array of circular floating porous elastic plates," Journal of Fluid Mechanics 900 (2020b). 